\title{
Experiences in accurately predicting time-dependent flows
}

\author{
Russell M. Cummings ${ }^{\mathrm{a}, *}$, Scott A. Morton ${ }^{\mathrm{b}}$, David R. McDaniel ${ }^{\mathrm{a}}$ \\ ${ }^{a}$ Department of Aeronautics, United States Air Force Academy CO 80840, USA \\ ${ }^{\mathrm{b}}$ Air Force Seek Eagle Office, Eglin Air Force Base, FL 32542, USA
}

\begin{abstract}
As computational fluid dynamics matures, researchers attempt to perform numerical simulations on increasingly complex aerodynamic flows. One type of flow that has become feasible to simulate is massively separated flow fields, which exhibit high levels of flow unsteadiness. While traditional computational fluid dynamic approaches may be able to simulate these flows, it is not obvious what restrictions should be followed in order to insure that the numerical simulations are accurate and trustworthy. Our research group has considerable experience in computing massively separated flow fields about various aircraft configurations, which has led us to examine the factors necessary for making high-quality time-dependent flow computations. The factors we have identified include: grid density and local refinement, the numerical approach, performing a time-step study, the use of sub-iterations for temporal accuracy, the appropriate use of temporal damping, and the use of appropriate turbulence models. We have a variety of cases from which to draw results, including delta wings and the F-18C, F-16C, and F-16XL aircraft. Results show that while it is possible to obtain accurate unsteady aerodynamic computations, there is a high computational cost associated with performing the calculations. Rules of thumb and possible shortcuts for accurate prediction of massively separated flows are also discussed.
\end{abstract}

\section{Contents}

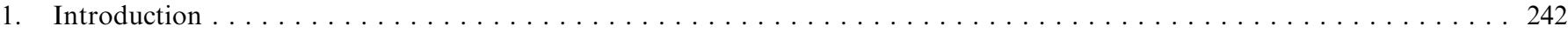

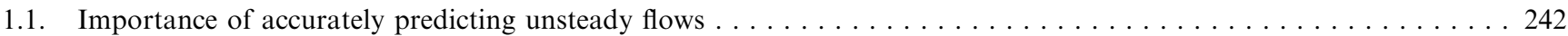

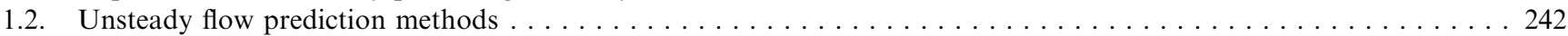

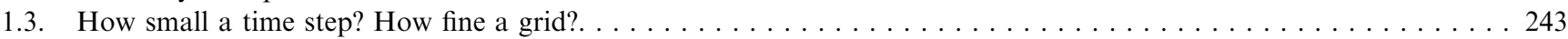

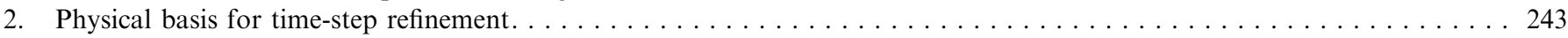

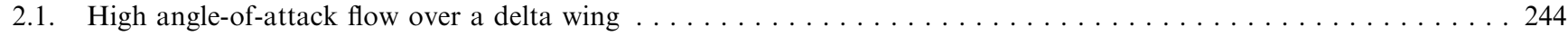

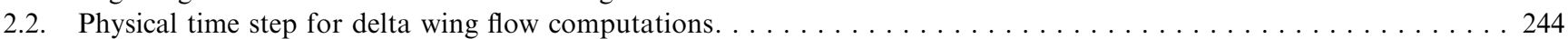

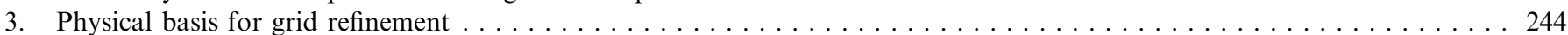

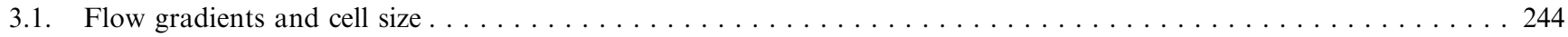

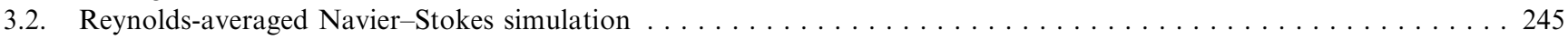

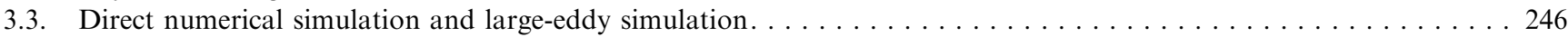

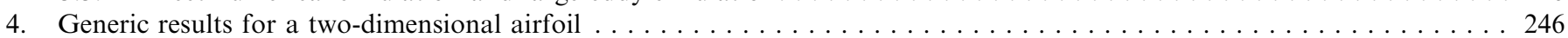

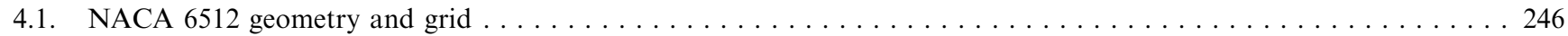

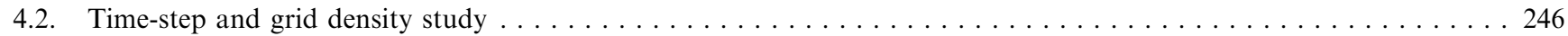

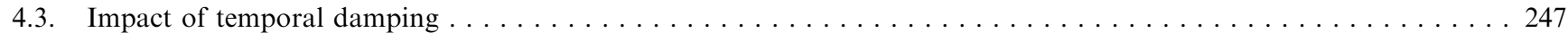

5. Grid refinement and time-step study for laminar flow over a delta wing . . . . . . . . . . . . . . . . 248

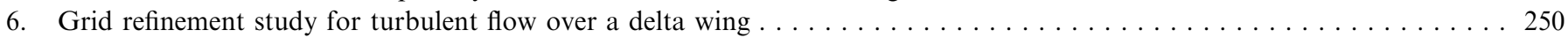




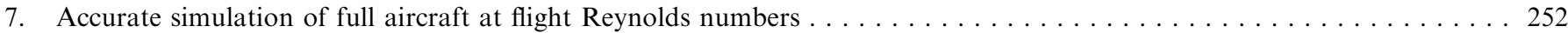

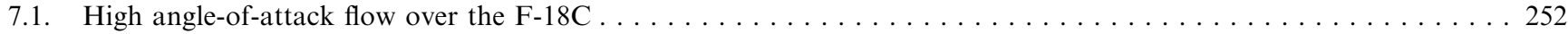

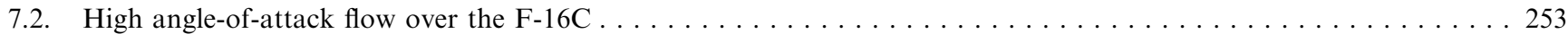

7.3. Medium angle-of-attack flow over the F-16XL $\ldots \ldots \ldots \ldots \ldots \ldots \ldots \ldots$

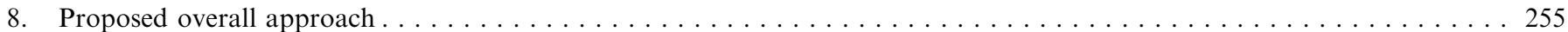

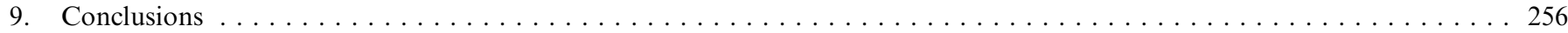

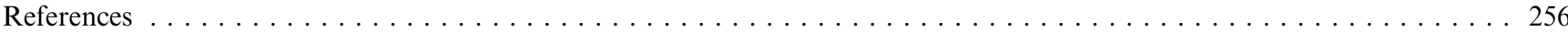

\section{Introduction}

While there has been a great deal of research into the development of accurate time-integration methods, very little work has taken place into quantifying the time steps necessary for accurate predictions of realistic unsteady flow fields. The importance of being able to predict timedependent flows goes far beyond the time-integration methods chosen, and requires detailed physical knowledge about the flow features being computed. Typically for unsteady flow simulations, researchers perform a grid sensitivity study for a steady flow case, choose a grid, and then (perhaps) perform some level of time-step study on that grid, an approach which may not correctly model the flow. In order to find a more universal method for performing grid and time-step studies, we will present a physical basis for choosing grids and time steps. The following sections will include a description of the importance of accurately predicting unsteady flow fields about complex configurations, as well as an overview of the common methods used to predict such flows. Finally, a discussion about the time steps required for these flow predictions will be undertaken.

\subsection{Importance of accurately predicting unsteady flows}

Many current military vehicles exhibit vortex-dominated flow fields. At a NATO air vehicle technology conference held in 2001, D. A. Lovell presented a review of "military vortices," where he discussed the importance of understanding the phenomena for the success of current and future aircraft. He classified vortex flows into three categories [1], "those designed into a vehicle to improve performance, those which cannot be avoided and whose adverse affects must be minimized, and those that were not expected to occur." He gives examples of many of these vortex-dominated flow fields: tip vortices on wings having low sweep, leading-edge extension vortices from the F-18 and F-16 aircraft, foreplanes on the Rafale, and flow over the MK-82 bomb (and other stores), to name just a few. Since the aerospace industry often concentrates on cruise conditions for optimization of commercial aircraft, these unsteady or vortical flow fields common in military aircraft are often not fully understood or able to be properly predicted. This is occurring at a time when the three largest US fighter development programs (F/A-18E/F, F-22, and $\mathrm{F}-35)$ incorporate twin tail configurations and high angleof-attack maneuvering. We believe it is clear that accurate prediction of unsteady flows for aircraft at non-cruise conditions is essential for future military aircraft development.

\subsection{Unsteady flow prediction methods}

Until recently, the state of simulation technology has not allowed for accurate prediction of vortex breakdown, and the unsteady flow downstream of breakdown, at flight Reynolds numbers. Because of this, researchers have spent time computing flows over simpler geometries, such as slender forebodies and delta wings, to improve their simulation capabilities. However, the advent of hybrid turbulence models may finally allow for accurate prediction of full aircraft flow fields at high incidence. While advances have taken place in areas such as grid generation and fast algorithms for solutions of systems of equations, computational fluid dynamics (CFD) has remained limited as a reliable tool for prediction of inherently unsteady flows at flight Reynolds numbers.

Current engineering approaches to prediction of unsteady flows are based on solution of the Reynoldsaveraged Navier-Stokes (RANS) equations. The turbulence models employed in RANS methods necessarily model the entire spectrum of turbulent motions. While often adequate in steady flows with no regions of reversed flow, or possibly exhibiting shallow or steady separation, it appears that RANS turbulence models are unable to accurately predict flows characterized by massive separation. Unsteady, massively separated flows are characterized by geometry-dependent and three-dimensional turbulent eddies. These eddies, arguably, are what defeat RANS turbulence models from predicting flows of such great complexity.

To overcome the deficiencies of RANS models for predicting massively separated flows, Spalart et al. [2] proposed detached-eddy simulation (DES) with the objective of developing a numerically feasible and accurate approach combining the most favorable elements of RANS models and large-eddy simulation (LES). The primary advantage of DES is that it can be applied at high Reynolds numbers, as can Reynolds-averaged techniques, but DES also resolves geometry-dependent, unsteady three-dimensional turbulent motions as in LES. Recently, Spalart et al. [3] proposed delayed detached-eddy simulation (DDES), which has the advantage of using a physical method for switching from RANS to LES (DES requires grid spacing to act as the switch). These hybrid models 
provide the tools that make the accurate prediction of massively separated flows possible.

\subsection{How small a time step? How fine a grid?}

In spite of the fact that the numerical tools exist for simulating massively separated flows, it is not clear how the tools should be used. How small should the time step be, and how fine should the grid be, in order to accomplish these calculations? These questions must be answered in a systematic way before researchers can confidently use the numerical tools that are now available.

Perhaps the first person to consider the question, "how much information is enough" was Harry Nyquist, a pioneer in the field of information theory. Nyquist was trying to determine the bandwidth requirements for transmitting information over telegraph wires and postulated that the bandwidth had to be twice the size of the amount of information being transmitted per unit time [4]. Extension of this concept led to the development of the famous Nyquist sampling theorem, which states that the sampling rate for accurate description of a transmitting wave has to be at least twice as fast as the frequency of the wave [5]. Examples of proper and improper sampling rates based on Nyquist's approach are shown in Fig. 1. A practical outcome of these early developments in information theory has been the unbelievable growth in telecommunications over the past decades, but we look to these concepts to find a minimum sampling rate (or maximum time step) for unsteady flow predictions. And while we are not "sampling" a transmitted wave, but rather computing an unknown unsteady flow, our restrictions will probably have to be even more confining than those of Nyquist.

In terms of establishing time-step limits for unsteady flow calculations, amazingly little has been published over the years. Research into determining time-step requirements has been so lacking, Spalart stated in [7] that, "space-time error balancing also leaves the most room for experimentation." Spalart [7] suggested a "rule of thumb"
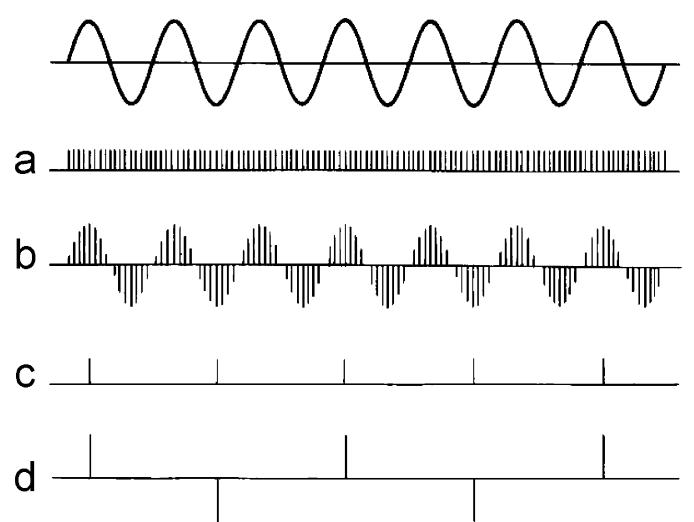

Fig. 1. Example of sampling rates: (a) a proper sampling rate for the given signal, (b) reproduced signal from the proper sampling rate, (c) too low a sampling rate for the given signal, and (d) reproduced signal from the improper sampling rate [6]. for DES computations, stating that a CFL number of approximately one was necessary for accurate prediction of large eddies, which was a requirement in both grid spacing and time step. Specifically, Spalart stated that $\Delta x_{0} / \Delta t=\leftarrow$ $U_{\max }$, where $\Delta x_{0}$ is the grid spacing in the LES focus region and $U_{\max }$ is the maximum velocity in that region (which could safely be assumed to be 1.5 to 2 times higher than $U_{\infty}$ ). Also, Spalart [7] and Schiff et al. [8] showed that at least five grid points (or cells) were required to model a large-scale flow feature correctly, meaning that $\Delta x_{0}$ needs to be at least five times less than the smallest flow structure resolved by LES.

Strelets [9] performed excellent simulations of massively separated flow fields using DES and determined that a nondimensional time step of $\Delta t^{*}=\theta .025\left(\Delta t^{*} \equiv \Delta t U_{\infty} / l\right.$, where $l$ is a characteristic length of the vehicle) was necessary for accurate prediction of massively separated turbulent flow fields. Görtz [10] found that a time step of approximately $\Delta t^{*}=\$ .006$ was required for accurate prediction of vortex breakdown over a delta wing at high angles of attack. Another study of high angle-of-attack flow over a delta wing by Schiavetta et al. [11] showed good results with a time step of $\Delta t^{*}=0.01$. All of these researchers showed excellent physical and numerical appreciation in their approaches, yet they all used somewhat different nondimensional time steps, leading us to believe that the choice of time step is quite complex and is affected by the algorithm, the grid being used, and the flow being simulated.

As we progress through the discussion about how to choose a grid and a time step for successful simulation of aerodynamic flows, we will follow the advice of Spalart [7], who said that, "gridding guidelines will be based on physical and numerical arguments, rather than on demonstrations of convergence to a "right" answer." We will not concentrate on comparisons with experimental data or theories; rather, we will see how choices made in the simulation effort impact the results.

\section{Physical basis for time-step refinement}

While it would be nice to have a simple rule for choosing the time step required for accurate prediction of flows over aircraft, the next section shows that the flow features often found around aircraft flying at high angles of attack do not allow for a single time step to be used for all cases. A physical discussion about delta wing flow features will lead to a choice of a maximum non-dimensional time step for high angle-of-attack unsteady flow predictions. Researchers performing numerical simulations of other flows would need to understand the time scales for their flow geometries in order to find adequate time steps, which may differ from the results presented here. In addition, knowledge about the numerical methods of the particular Navier-Stokes solver being used is important as well. Solutions for the following studies were computed with the Navier-Stokes solver Cobalt developed by Cobalt Solutions, LLC [12]. 
Cobalt solves the unsteady, three-dimensional, compressible Navier-Stokes equations on a hybrid unstructured grid.

\subsection{High angle-of-attack flow over a delta wing}

As an example of determining the physically correct time step for a flow computation, we will use high angle-ofattack flow over a delta wing as an example. A large number of researchers have described the unsteady nature of various flow features found in high angle-of-attack delta wing flows, and a good summary of their findings was put together by Schiavetta et al. [11]. The summary of these results is outlined in Table 1 and Fig. 2, where flow features such as the helical mode instability, vortex shedding, and vortex breakdown oscillation are shown with their corresponding Strouhal numbers $\left(S t \equiv f / U_{\infty}\right)$. Notice that the oscillation frequencies for the various flow features vary by several orders of magnitudes, which makes accurate prediction of these flows even more complicated.

\subsection{Physical time step for delta wing flow computations}

Based on the results presented for vortical flow fields (as shown in Table 1 and Fig. 2), the highest Strouhal number likely in this high incident flow approaches $S t \approx 20$, which would be found in a shear layer instability. Assuming that the flow being computed contains a shear layer, and further utilizing the Nyquist sampling rate of at least twice the frequency of interest, a computation for such a flow as shown in Fig. 2 should have a non-dimensional time step of $\Delta t^{*} \leqslant 0.025$, a value which was used in the initial DES computations of Strelets [9].

Table 1

Frequencies corresponding to important unsteady features of vortical flows over delta wings [11]

\begin{tabular}{ll}
\hline Phenomenon & Strouhal number \\
\hline Helical mode instability & $1-2$ \\
Shear layer instabilities & $8-10$ and higher \\
Vortex shedding-TE & $\approx 8$ \\
Vortex shedding_high $\alpha$ & $0.2-0.5$ \\
Vortex breakdown oscillation & $0.01-0.08$ \\
\hline
\end{tabular}

This validates the earlier time-step results for similar flow fields [8-10], but also points out that there should be a general "rule of thumb" for the choice of time step: the time step should be determined by the temporal aspects of the flow feature ( $s$ ) of interest in the computation. For example, Table 1 shows Strouhal numbers ranging from $0.01 \leqslant S t \leqslant 10+$, so it might be possible to use a much higher time step than $\Delta t^{*}=\$ .025$, depending on the flow features of interest. However, our research has shown that the flow features described in Table 1 are often interrelated, meaning that in order to accurately predict the vortex breakdown oscillation (where $S t \approx 0.01$, requiring a time step as high as $\Delta t^{*}=50$ ) the shear layer instability must also be accurately modelled first (requiring a time step as low as $\Delta t^{*} \leqslant 0.025$ ). We use $\Delta t^{*}=\theta .01$ as a starting point for our calculations. If you are not sure what the time step should be, then taking the time to perform a time-step sensitivity study is worth the effort.

\section{Physical basis for grid refinement}

While it is essential to find an appropriate time step for accurate prediction of time-dependent flows, it is also important to know how fine to make the grid. While it may be possible to simply refine the grid until all appropriate flow features emerge, this approach can be computationally quite expensive, and in fact may not lead to more accurate flow predictions. A physical basis for choosing the appropriate grid is important for accurate flow prediction.

\subsection{Flow gradients and cell size}

What makes a given numerical prediction spatially accurate? Of course this is determined both by the numerical methods being used, the grid itself, and the flow being predicted, as can be demonstrated with a flow shown in Fig. 3. Assume that the numerical prediction method uses a finite difference approach with a second-order accurate, one-sided first derivative, where the leading truncation term is of the form: $\Delta x^{2} \partial^{3} f / \partial x^{3}$ (where $f$ is some physical property such as pressure, density, temperature, or velocity). What will make the truncation term small (and therefore lead to an accurate flow prediction)?

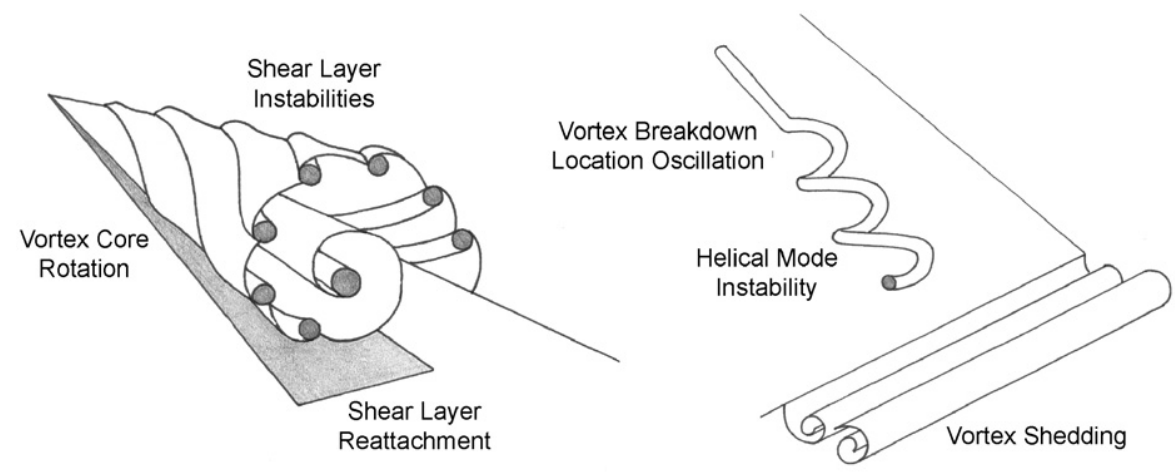

Fig. 2. Types of unsteadiness in delta wing flow [11]. 
If $\partial^{3} f / \partial x^{3} \rightarrow 0$ (small flow gradient region of Fig. 3) then $\Delta x$ can be "large" and still retain accuracy. If $\Delta x \rightarrow 0$ then $\partial^{3} f / \partial x^{3}$ can be "large" (large flow gradient region of Fig. 3) in order to retain good accuracy. Alternatively, a limit of the error can be obtained by finding appropriate levels of grid spacing to correspond to various flow gradients.

These results correspond directly to the various flow structures described in Fig. 2 and Table 1. Knowledge about the frequencies of the various types of flow unsteadiness is essential, but so is knowledge about the size and location of the various flow structures. Obviously, the vortices shown in Fig. 2 require appropriate levels of grid support (at least five cells across the width of the vortex, as well as five cells across the width of secondary vortices and separation locations as well), but if the shear layer instability is also being simulated, the cell size might need to decrease by an order of magnitude or more in that region. Likewise, many computations do not concentrate

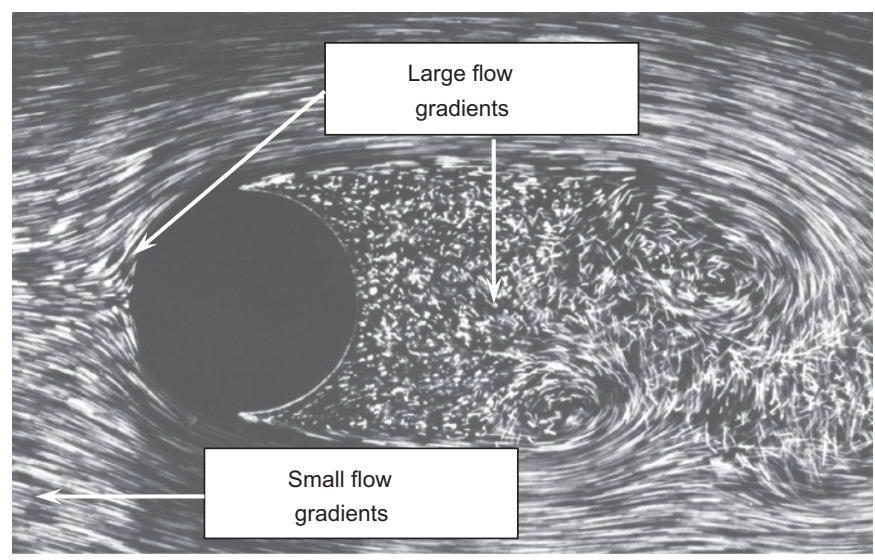

Fig. 3. Relationship between flow gradients and grid size [13]. on the wake region of a delta wing, but an accurate prediction may require that the trailing-edge wakes also be predicted well. This leads to another rule of thumb in accurate flow prediction: it is essential to understand the spatial aspects of the fluid dynamic processes being simulated in order to obtain accurate flow predictions. The situation can even be made more difficult if dynamic motion is involved in the simulation, since the fluid structures can also be reacting to unsteadiness of the boundary conditions.

\subsection{Reynolds-averaged Navier-Stokes simulation}

In addition to knowledge about various flow structures being resolved in the numerical simulation, knowledge about the boundary layer (including the state: laminar or turbulent) is also essential when using RANS simulations. Since RANS requires modelling of all turbulence scales (both spatial and temporal), the grid support required to accomplish good simulations must be understood. Fig. 4 shows the velocity variation in a typical turbulent boundary layer $\left(u^{+}\right.$is the velocity within the boundary layer and $y^{+}$the distance from the surface in wall units). The upper portion of Fig. 4 shows the velocity profile on a linear length scale, and the lower portion shows the same velocity variation on a logarithmic scale. The lower portion shows that each region of the boundary layer has a different velocity variation, which requires that each layer needs appropriate grid resolution in order for turbulence models to work appropriately. Some rules of thumb commonly employed when simulating a boundary layer include that the first grid point away from the surface should be located at $y^{+} \approx 1$, with at least two or three grid points within the viscous (or laminar) sublayer, and

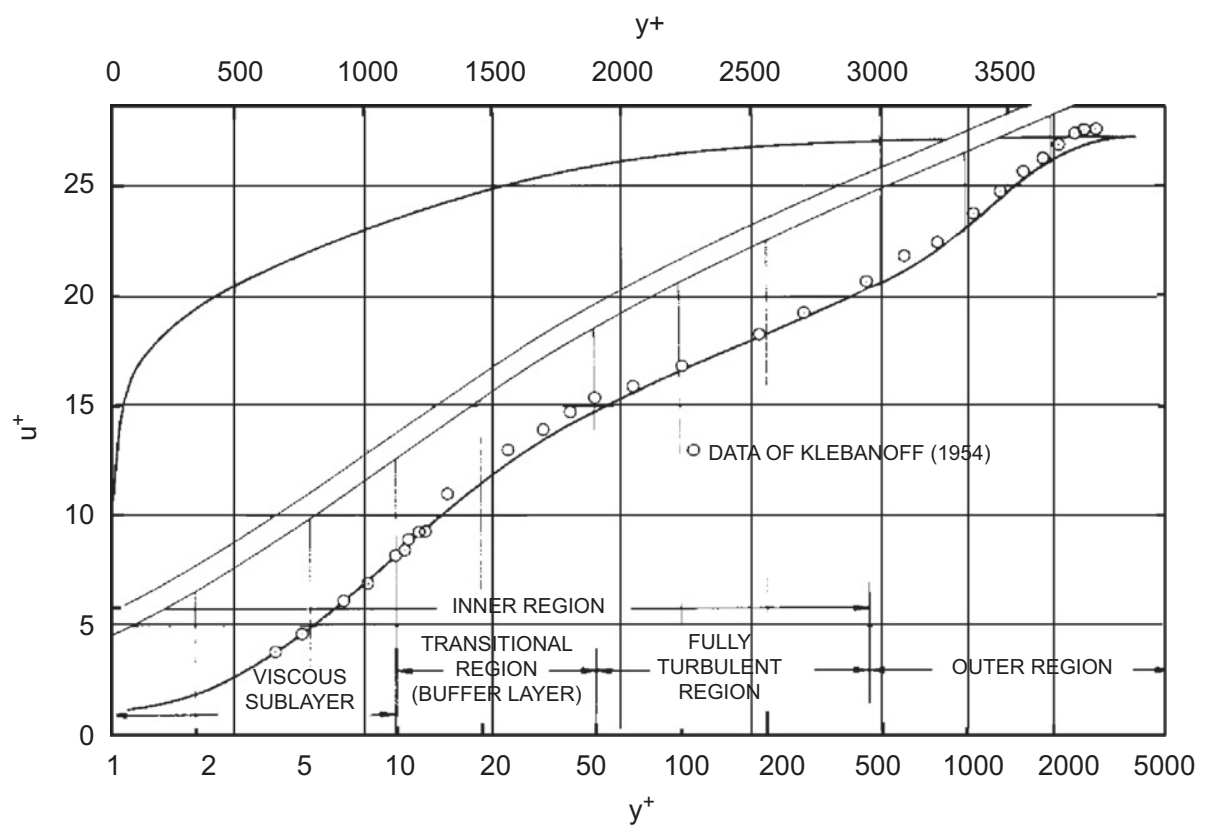

Fig. 4. Velocity profile throughout a turbulent boundary layer [14]. 
a maximum grid-stretching ratio of approximately 1.25. These rules produce at least 20 grid points within the boundary layer, another common rule for accurate prediction of a boundary layer using RANS methods.

\subsection{Direct numerical simulation and large-eddy simulation}

LES and DES require special care in creating grids and choosing time steps. Understanding the capabilities of subgrid-scale turbulence models is essential in understanding the needs for resolving large eddies.

Since direct numerical simulation (DNS) resolves all levels of turbulence scales, it requires knowledge about the spatial and temporal scales of turbulence. The Kolmogorov turbulence microscales for length $(\eta)$ and time $(\tau)$ are given by

$\eta=\left(\frac{v^{3}}{\varepsilon \psi}\right)^{1 / 4}, \psi \tau=\left(\frac{v}{\varepsilon}\right)^{1 / 2}$

where $v$ is the kinematic viscosity and $\varepsilon$ the turbulence dissipation rate per unit mass [15]. Since these relationships define the spatial and temporal scales of turbulence, they therefore set the requirements for grid spacing and time steps for DNS. For example, the grid spacing must be less than the turbulence length scale and the time step must be less than the turbulence time scale. This leads to a threedimensional calculation requiring $R e^{9 / 4}$ grid points and the number of time steps is proportional to $\operatorname{Re}^{3}$ [16]. These requirements result in massive computer storage and long run times in order to perform DNS calculations, especially as the Reynolds number is increased.

LES can be seen as a compromise between DNS and RANS. The basis of LES is that the flow is decomposed into small and large length scales. The small scales are modelled while the large scales (large eddies) are solved for numerically. The cost of computation is greater than RANS, but less than DNS. Since the small scales are modelled, the Reynolds number restriction of DNS is eased a great deal.

\section{Generic results for a two-dimensional airfoil}

In an attempt to outline some of the issues that are important for accurate prediction of time-dependent flows, a basic study for a two-dimensional airfoil was undertaken. Specifically, the NACA 6512 airfoil was used to perform a fairly comprehensive time step, grid, and numerical algorithm properties study.

\subsection{NACA 6512 geometry and grid}

A two-dimensional airfoil with high camber $(6 \%$ of chord) and moderate thickness ( $12 \%$ of chord) was selected to perform a grid sensitivity study and calculate the laminar vortex shedding that takes place from the trailing edge of the airfoil at low Reynolds numbers, as shown in
Fig. 5. The airfoil flow field was computed at a Reynolds number $R e_{c}=4.0 \times 10^{5}$ and at an angle of attack $\alpha=\theta^{\circ}$. Under low-Reynolds conditions, Karman vortex shedding takes place in the vicinity of the trailing edge of the airfoil, establishing a well-known unsteady flow with experimentally measured Strouhal numbers that vary depending on the angle of attack and Reynolds number [17]. In fact, the experimental data show that transition would take place under the conditions shown here, but the results serve the purpose of finding a grid and time step that resolve the flow features.

A joint time-step/grid resolution study will be shown, which was performed on three grids, with the characteristics shown in Table 2. The three grids were created to give a wide variation of cells in the flow field (especially in the wake region of the airfoil), with the number of cells ranging from approximately 15,000 to nearly 150,000 for the coarse to fine grids, respectively. All grids had nearly identical grid spacing near the surface, with an average initial spacing of $y_{\text {avg }}^{+} \approx \theta .7$, which is certainly adequate for a laminar boundary layer at low Reynolds number. The airfoil was simulated for a free-stream Mach number $M_{\infty}=\theta .1$ and a chord-based Reynolds number $R e_{c}=4.00 \times 10^{5}$.

\subsection{Time-step and grid density study}

A time-step/grid density study was performed with the airfoil grids presented in Table 1 . The grids were run at five or six time steps (depending on how many reductions in the time step were required in order to reach time-step "convergence"). The time steps used were $\Delta t=\theta .00040$, $0.00020,0.00010,0.00005,0.000025$, and $0.0000125 \mathrm{~s}$, which correspond to non-dimensional time steps of

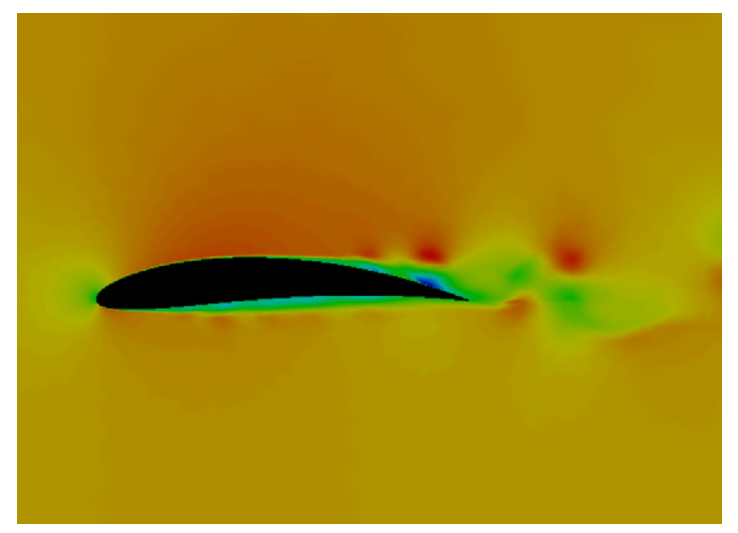

Fig. 5. Vortex shedding from the trailing edge of a NACA 6512 airfoil.

Table 2

Comparison of NACA 6512 airfoil grids

\begin{tabular}{llcc}
\hline Grid name & Number of points & Number of faces & Number of cells \\
\hline Coarse & 11,101 & 25,990 & 14,897 \\
Medium & 25,648 & 62,771 & 37,131 \\
Fine & 78,907 & 220,513 & 141,615 \\
\hline
\end{tabular}


$\Delta t^{*} \equiv \Delta t U_{\infty} / c=\$ .01325, \quad 0.00662, \quad 003312, \quad 0.001656$, 0.000828 , and 0.000414 , respectively. These values were initially chosen based on our "rule of thumb" for aerodynamic flows, such as those described in Table 1, which are usually modelled with a non-dimensional time step of $\Delta t^{*} \approx 0.01$ (shown on Fig. 6). This non-dimensional time step meets the Nyquist sampling rate requirement mentioned earlier and usually aids the researcher in performing a reasonable time-step study.

Each solution was run for the same amount of physical time in second-order accurate mode, in this case $t=\theta .4 \mathrm{~s}$, which means that for $\Delta t=\theta .00040 \mathrm{~s}$ cases the solution was run for 1000 iterations in time, for $\Delta t=\theta .00020 \mathrm{~s}$ cases the solution was run for 2000 iterations, etc. Three Newton sub-iterations were used for all cases, and the damping levels were set to default values (damping levels will be discussed later). The simulations had been initiated with 500 iterations of first-order accurate simulations (in order to reach a converged flow field), and then each case was run from the same steady flow solution. Once the solutions were obtained, time-accurate normal force variations were used to perform a power spectrum density (PSD) analysis, which resulted in the wave numbers shown in Fig. 6.

Notice that each of the grids shows a reduction in the wave number (assumed here to be the inverse of the Strouhal number), as the time step is reduced enough (say to $\Delta t=\$ .00010 \mathrm{~s}$ for the coarse grid). As the grid is refined, the time step must be reduced further in order to reach time-step convergence, until two grids converge to the same wave number, in this case when the medium and fine grids reach a wave number of approximately 0.6 . Note that this wave number corresponds to a Strouhal number of $S t \approx 1.67$, which corresponds to the experimental value for vortex shedding. In this case the results show that either the medium grid (at $\Delta t=\theta .000025 \mathrm{~s}$ ) or the fine grid

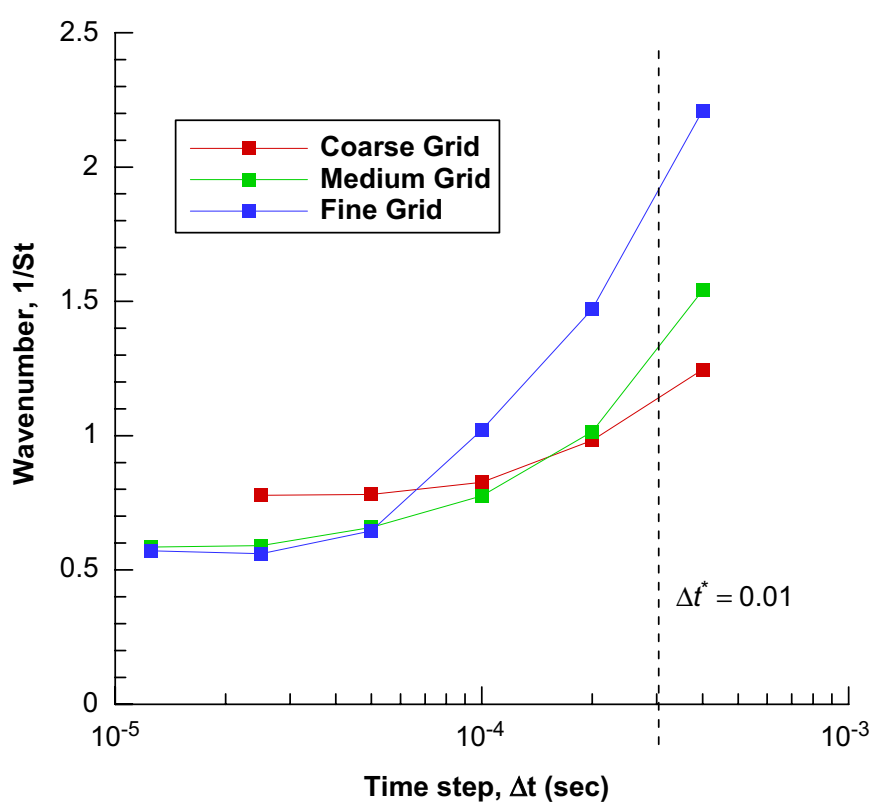

Fig. 6. Time-step and grid convergence study for NACA 6512 airfoil. (at $\Delta t=8.000050 \mathrm{~s}$ ) can simulate a reasonably accurate vortex shedding from the airfoil, and the user would decide between them based on computer resources or possibly other accuracy considerations. Another note of caution can be found in these results: each grid required a different time step to obtain time independence, a fact that should make those who do not perform a joint time-step/grid density study wary.

\subsection{Impact of temporal damping}

Another important factor in the accurate prediction of time-dependent flows is the numerical damping that is often added to the time-integration schemes. Damping is often required in order to maintain temporal stability, but too much damping can degrade the accuracy of the simulation. As with the time-step study, it is important to determine the appropriate levels of temporal damping being used, and to use the least amount of damping feasible.

An example of such a temporal damping study is shown in Fig. 7 for the NACA 6512 airfoil. In this case, the medium grid was used with a time step of $\Delta t=\theta .000050 \mathrm{~s}$ (a case that gave good results for the time-step study shown in Fig. 6) and three Newton sub-iterations; all solutions were started from the same converged time-accurate computation and run for 8000 iterations. The advection damping coefficient (which damps the inviscid fluxes) was varied from 0.050 to 0.005 while holding the diffusion damping coefficient (which damps the viscous fluxes) constant at 0.01 . At the largest value of advection damping coefficient $(0.050)$ the results showed the expected highfrequency oscillations, but a lower frequency was also evident (note the decrease in the normal force as a function of time). As the temporal damping was decreased (say from

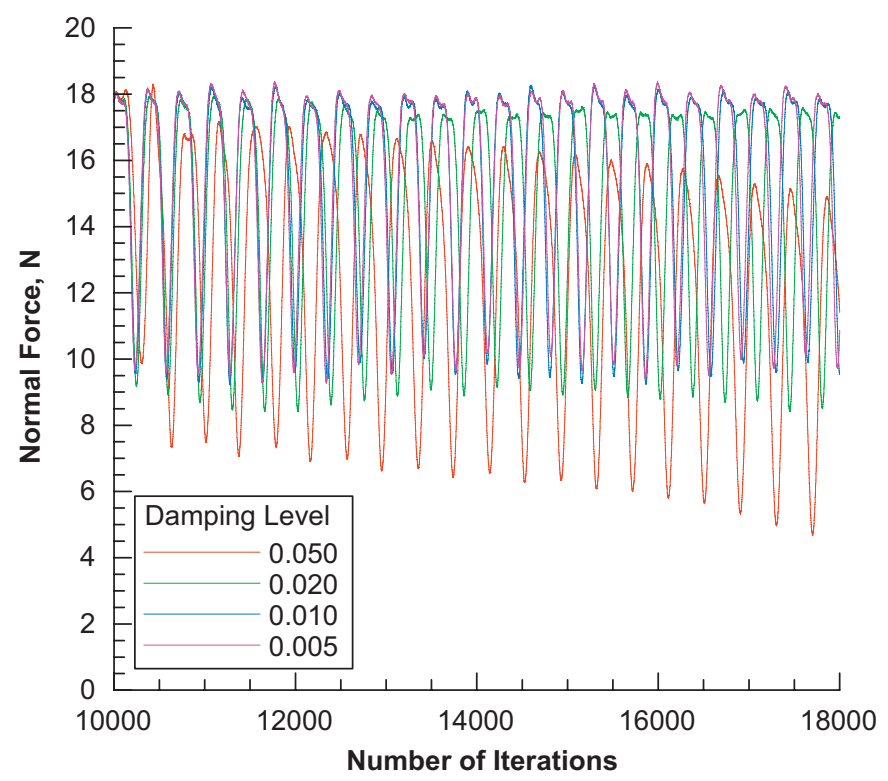

Fig. 7. NACA 6512 airfoil force oscillations with various levels of temporal damping. 
0.050 to 0.020 ) the lower frequency oscillation was less evident, but a change in the high-frequency Strouhal number also took place. Not until the temporal damping was reduced to 0.010 and 0.005 did two identical time integrations take place (in both amplitude and frequency). The NACA 6512 airfoil flow calculations would therefore have to be performed with the temporal damping at 0.010 or lower in order to achieve the best accuracy at this timestep and sub-iteration level. Lower time steps or more subiteration levels would make a higher damping level equally as accurate.

\section{Grid refinement and time-step study for laminar flow over a delta wing}

The previous descriptions show the need for performing a grid convergence and time-step study whenever computing unsteady flow fields. While there are probably numerous ways to achieve such a study, we have developed a systematic method for determining the time step and sub-iteration levels required for accurately computing time-dependent flow fields. The method will initially be described in this section, and then used in subsequent sections on more complex aircraft.

A flat-plate delta wing with a leading-edge sweep of $70^{\circ}$ and a $25^{\circ}$ bevel on the lower surface was investigated in the US Air Force Academy $38 \mathrm{~cm} \times 110 \mathrm{~cm}$ free-surface water tunnel. A companion numerical simulation was also performed [18]. The wing had a chord length of $298 \mathrm{~mm}$ and was set at an angle of attack $\alpha=35^{\circ}$, and was used for a study to determine the practicality of using periodic suction and blowing as a flow control method. The experimental free-stream velocity was $0.126 \mathrm{~m} / \mathrm{s}$, and the corresponding root-chord Reynolds number was $R e_{c}$ $=4.07 \times 10^{4}$. The free-stream Mach number for the computations was set to $M_{\infty}=\theta .1$, with the free-stream pressure and temperature chosen to match the Reynolds number of the experiment.

In order to determine the appropriate grid density and time step for numerical simulations, a study was carried out for the highly unsteady flow field caused by vortex breakdown above the delta wing. Fig. 8 shows the variation of normal force on the delta wing as a function of time step for the coarse grid at three time steps, $\Delta t=\theta .020,0.010$, and $0.005 \mathrm{~s}$ (a solution was also obtained for $\Delta t=\theta .0025$, but is not shown for clarity). As can be seen, the vortex breakdown has a high-frequency content as well as a second, lower frequency variation with time. Also, it should be obvious that while the three time steps yield somewhat similar results, the frequency content for each is not exactly the same. We have resorted to using PSD analysis for these types of computations to determine the magnitude of frequencies seen in Fig. 8.

The PSD of the coarse grid solutions were obtained using MATLAB and plotted in Fig. 9a. Each of the four time steps used produces a different primary frequency (shown as the wave number). Clearly, the dominant

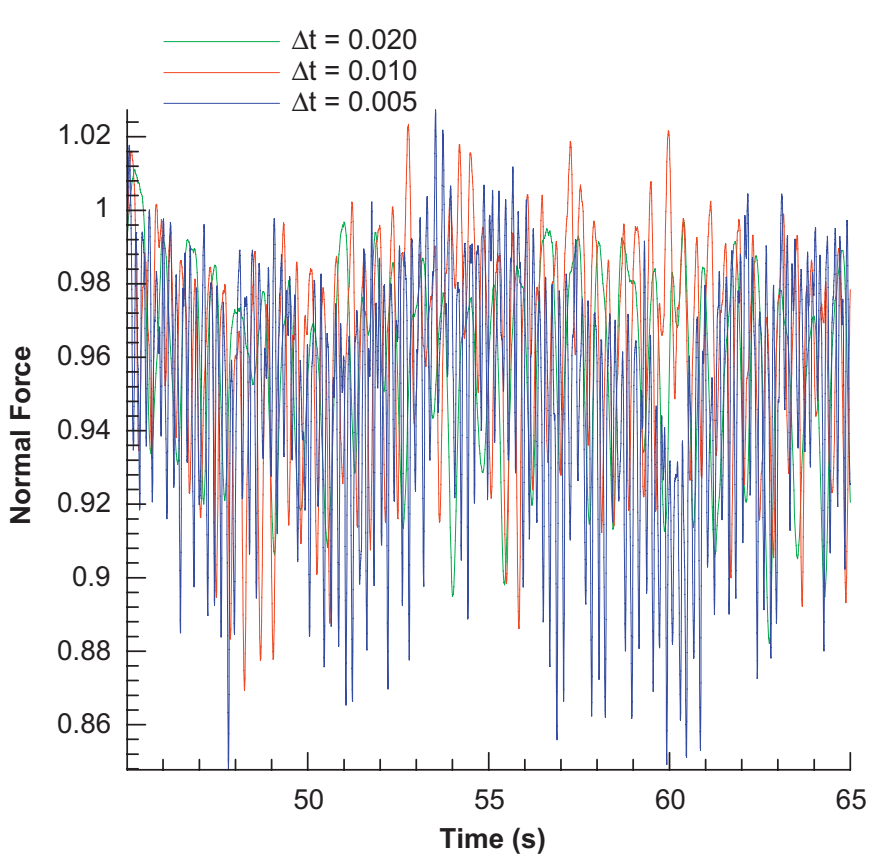

Fig. 8. Coarse grid normal force variation with time [18].

unsteady feature of the flow (the vortex breakdown) is not properly resolved if each time step yields a different primary frequency. In order to determine whether or not a "converged" time step is being approached, the wave number for each time step is plotted against the logarithm of the time step in Fig. 9b. While the incremental change in wave number decreases with a decrease in time step, the appropriate time step has probably not been attained (with the best wave number computed being approximately 10 ). In addition to the lack of time-step convergence, the coarseness of the grid probably will not allow for an appropriate vortex frequency to be modelled.

A similar study was then conducted using a finer grid. Four time steps (starting with values comparable to those used for the coarse grid) are shown in Fig. 10a. The computations were all performed for the same physical time $(10 \mathrm{~s})$ by varying the number of iterations for each time step (2500 iterations for $\Delta t=0.004 \mathrm{~s}, 5000$ iterations for $\Delta t=\theta .002 \mathrm{~s}$, etc.), and each computation was completed with two Newton sub-iterations. The calculations were carried out so that at least 10 full cycles of the frequencies of interest were included in the results in order to facilitate power spectrum analysis. The resulting wave numbers show an improvement over those resulting from the coarse grid, with the lowest wave number (highest frequency) of approximately 3, as shown in Fig. 10b. Details of the frequency spectrum are also much more complex for the fine grid compared to the coarse grid, as evidenced by the multiple power spikes in the vicinity of the primary frequency (compare Fig. 10a with Fig. 9a). These solutions clearly show a second, higher frequency fluctuation in the flow field. For $\Delta t=\theta .004 \mathrm{~s}\left(\Delta t^{*}=\theta .45\right)$ the primary frequency takes place at a wave number of approximately 23 , and the second frequency takes place 
a

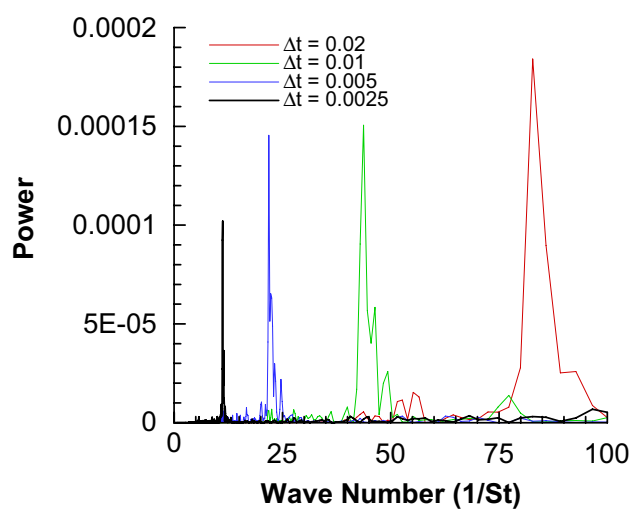

b

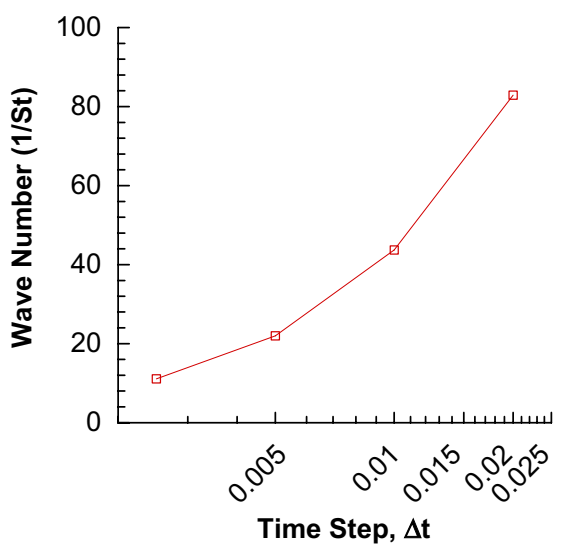

Fig. 9. Power spectrum density and wave number variation for coarse grid [18]: (a) power spectrum density and (b) wave number.

a

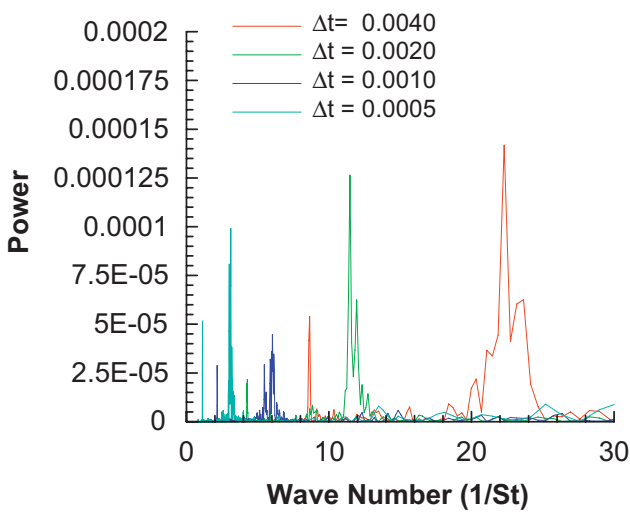

b

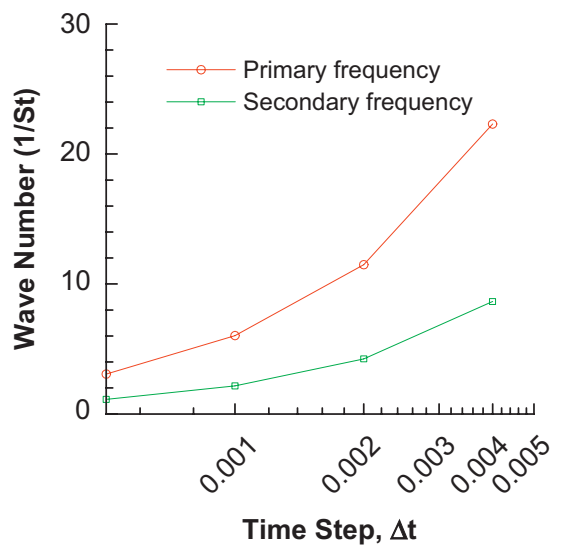

Fig. 10. Power spectrum density and wave number variation with time step for coarse grid [18]: (a) power spectrum density and (b) wave number.

at a wave number of approximately 9 . For $\Delta \mathrm{t}=\theta .001 \mathrm{~s}$ $\left(\Delta t^{*}=0.11\right)$ the primary frequency takes place at a wave number of approximately 6 , and the second frequency takes place at a wave number of approximately 2 , vastly different values than for the higher time step. Similar results can be seen for all four cases shown in Fig. 10a.

These results are consolidated and shown as a function of time step in Fig. 10b. As can be clearly seen, both the primary and secondary frequencies are converging to a constant value as the time step decreases. In addition to both variations decreasing asymptotically, the difference between wave number variations decreases as the time step decreases. While it is not obvious that the two frequencies will asymptote to the same value, it is possible. However in this case the secondary frequency was found to correspond to oscillations in the breakdown of the secondary vortex [18]. Also, while a converged time step has not been attained, it is clear that the appropriate wave number is somewhere in the range of 0.5 (which corresponds to a Strouhal number $S t=2$ ).
An additional temporal study was then performed to determine the effect of number of Newton sub-iterations on the solution. Fig. 11 shows the wave number variation for five Newton sub-iteration levels $\left(n_{\text {sub }}=4,2,3,4\right.$, and 5), all at a time step of $\Delta t=0.00005 \mathrm{~s}\left(\Delta t^{*}=\theta .006\right)$. Once again, two frequencies can be seen for each time step (for $n_{\text {sub }}=4$ the primary frequency takes place at a wave number just below 9, with a second frequency evident above 3). This is repeated for all four Newton sub-iteration levels shown. Similar to the results seen in Fig. 10, the wave number for both primary and secondary frequencies decreases with increasing levels of Newton sub-iterations. In fact, the secondary frequency seems to have nearly converged, while the primary frequency is very nearly converged at $n_{\text {sub }}=4$. While neither of the studies (time step and Newton sub-iteration) have been shown to be completely converged, the essential features of the flow field are appropriately modelled for the purposes of this comparison with a time step of $\Delta t=0.0001 \mathrm{~s}\left(\Delta t^{*}=0.011\right)$ and a Newton sub-iteration level of $n_{\text {sub }}=\xi$ using the fine grid. Certainly, slightly improved solutions would be 


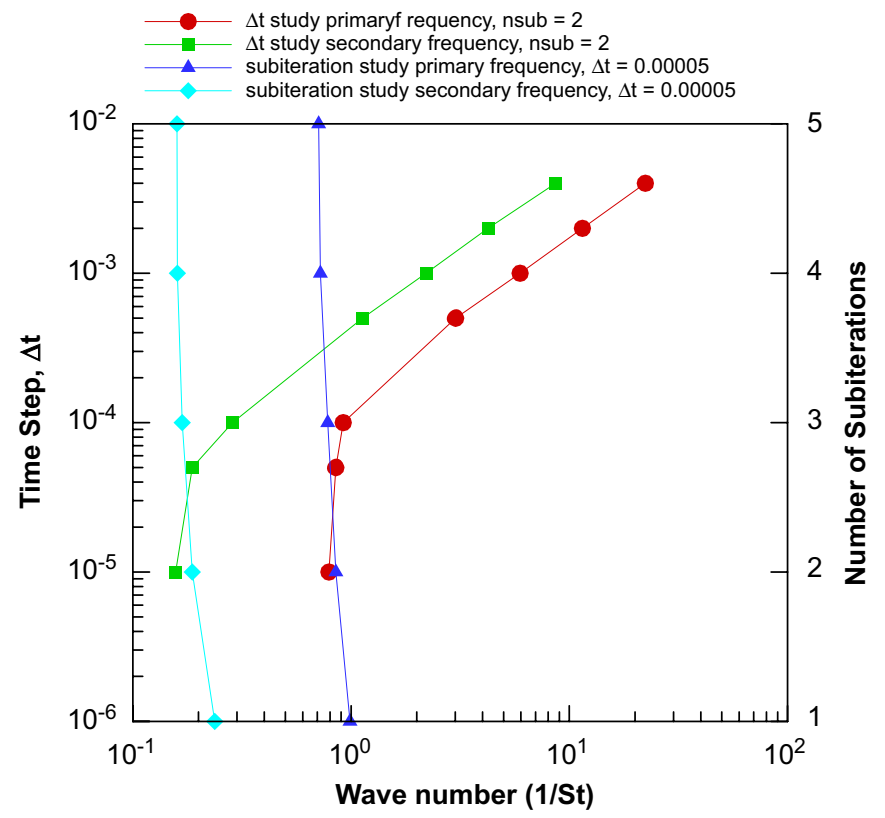

Fig. 11. Wave number variation with Newton sub-iteration level for fine grid [18].

obtained by using $\Delta t=\theta .00005 \mathrm{~s}$ and $n_{\text {sub }}=4$, but very little essential information would be gained for the added computational cost.

While this example was one of our first attempts at a formalized time-step study, a number of important concepts were learnt. First, a time-step study makes no sense when separated from a grid resolution study, since the fineness of the grid determines the flow features as much as the time scale. Second, it is not easy to look at force variations and determine when two computations are similar or different in terms of frequency content, so a power spectrum analysis helps a great deal in determining details of the flow. Finally, time-step accuracy is also a function of time-integration method accuracy, and must be researched in addition to the actual time step used. These results are now taken and applied to more complex configurations.

\section{Grid refinement study for turbulent flow over a delta wing}

The delta wing model in this study has a $70^{\circ}$ sweep angle and root chord $c=950 \mathrm{~mm}$. The delta wing has a span $b=691.5 \mathrm{~mm}$ at its trailing edge, is $20 \mathrm{~mm}$ thick, and is beveled on the windward side at an angle of $15^{\circ}$ to form sharp leading edges (see Fig. 12). The experimental data presented in this paper was acquired in ONERA's $1.4 \mathrm{~m} \times 1.8 \mathrm{~m}$ subsonic wind tunnel $(\mathrm{F} 2)$ at test conditions of $\alpha=27^{\circ}$ and $U_{\infty}=24 \mathrm{~m} / \mathrm{s}\left(R e_{c}=4.56 \times 10^{6}\right)$. Due to the relative symmetry of the flow field over the leeward surface of the delta wing, only the portside flow field was examined. Details of the model, the wind tunnel, and LDV system, as well as the computational study are specified in [19].

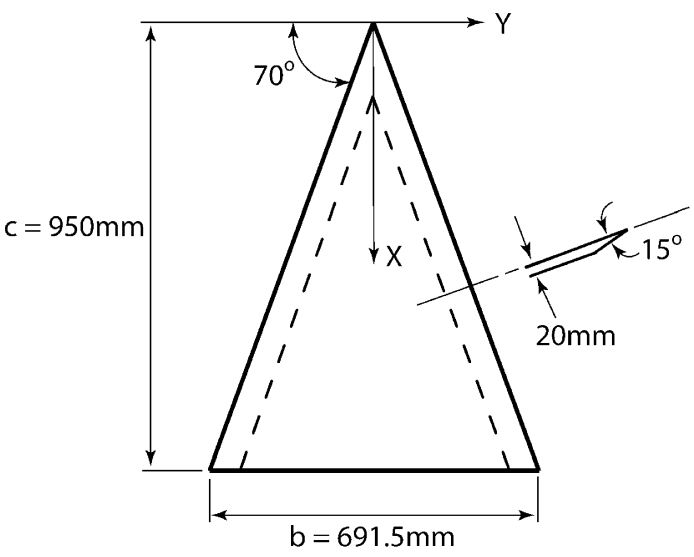

Fig. 12. Delta wing model with $70^{\circ}$ leading-edge sweep [19].

Table 3

Grids used in $70^{\circ}$ leading-edge sweep delta wing computations [19]

\begin{tabular}{llc}
\hline Grid & Name & Number of cells (millions) \\
\hline 1 & Coarse & 1.2 \\
2 & Medium & 2.7 \\
3 & Fine & 6.7 \\
4 & Very fine & 10.7 \\
5 & AMR & 3.2 \\
\hline
\end{tabular}

Four semi-span grids were created for the delta wing (as described in Table 3) of 1.2 (Grid 1), 2.7 (Grid 2), 6.7 (Grid 3), and 10.7 million cells (Grid 4). Each grid in the series is refined in all three coordinate directions by a factor of $1 / \sqrt{2}$ from the previous grid in the series. Results from the grids were presented in [19], including a fifth grid created with adaptive mesh refinement (AMR) that had 3.2 million cells (Grid 5).

All of the grids in this study consist of an inner region of approximately 13 layers of prisms for the boundary layer, with a wall normal spacing in viscous wall units less than 1 $\left(y^{+} \leqslant 1\right)$, and an outer region of tetrahedra. The prism dimensions on the surface were a factor of approximately 200 times larger than the wall normal dimension for all grids.

Pirzadeh's AMR method [20] was applied to the ONERA delta wing configuration in this study. A steadystate flow solution was computed for a grid with surface resolution between the coarse and medium grids described above (Grids 1 and 2, respectively), and then was used to create an AMR grid by eliminating all cells within an isosurface of vorticity at a particular level. The grid was then grown inside the iso-surface with a scale factor of 0.5 . This procedure was performed twice to create a vortex core and shear layer with one fourth of the cell sizes (in all coordinate directions) of the original grid. The new grid was then used to compute unsteady DES for the flow field.

Figs. 13a-d depict crossplanes of the very fine and AMR grids (Grids 4 and 5, respectively), at four chord-wise stations, $x / c=\theta .53,0.63,0.74,0.84$. It is apparent from 

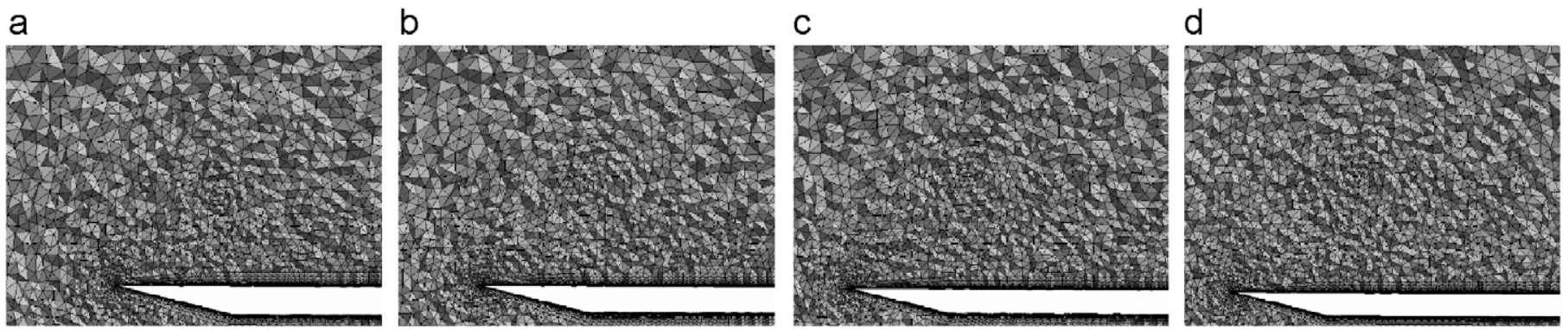

Fig. 13. Crossplanes of Grid 4 (10.7 million cells) at four chordwise stations [19]: (a) $x=400 \mathrm{~mm}$; (b) $x=\$ 00 \mathrm{~mm}$; (c) $x=600 \mathrm{~mm}$ and (d) $x=\not 00 \mathrm{~mm}$.
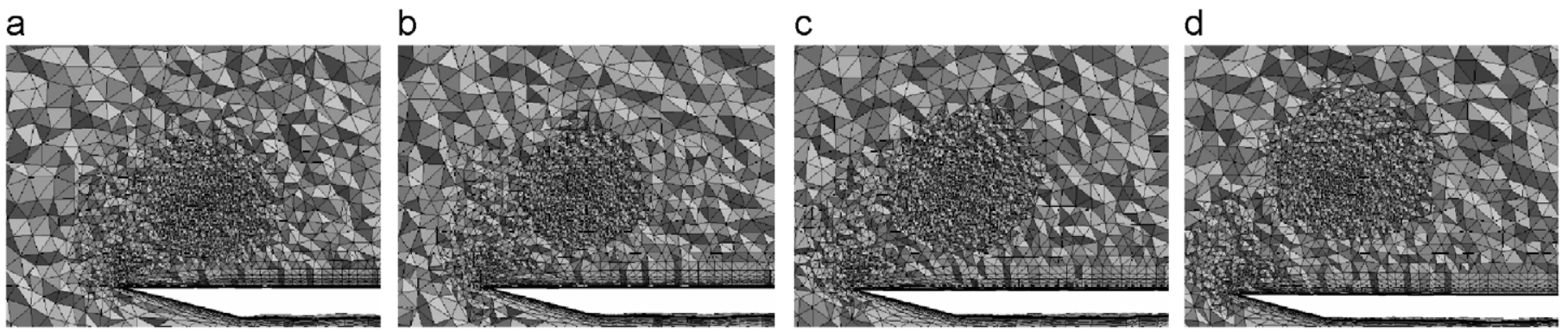

Fig. 14. Crossplanes of Grid 5 (3.2 million cells) at four chordwise stations [19]: (a) $x=400 \mathrm{~mm}$; (b) $x=500 \mathrm{~mm}$; (c) $x=600 \mathrm{~mm}$ and (d) $x=\$ 00 \mathrm{~mm}$.

Figs. 13a and $\mathrm{b}$ that a consistent grid refinement has occurred with very little emphasis on the vortex core or shear layer. In contrast, the AMR grid (Grid 5) depicted in Fig. 14 shows a concentration of points in the vortex core and leading-edge shear layer regions with cell sizes smaller than even Grid 4 of Fig. 13. It should be noted that the shear layer loses resolution for the chord-wise stations of 0.74 and 0.84 . Fig. 15 depicts a downward look on a plane passing through the surface of the delta wing grid. The left side is the very fine grid (Grid 4) and the right side is the AMR grid (Grid 5). It is apparent that Grid 4 has refined cells outboard of the leading edge in a region that, arguably, has little impact on the solution. It is also clear that the trailing-edge region is much more refined in Grid 4, making it superior to Grid 5 for resolving the unsteady wake region emanating from the blunt trailing edge. Grid 5 , however, has approximately one third the cells of Grid 4, which makes Grid 5 computationally more efficient for a similar level of simulation capability.

Typical simulations were run for 10,000 iterations, starting from free-stream conditions, and time averages were computed starting after the 2000th iteration to eliminate transients. Figs. 16a-e show a top view of the delta wing for the five grids discussed previously. An isosurface of vorticity magnitude colored by the spanwisevorticity component is displayed for each of the grids (all vorticity components and iso-surfaces were defined the same for these figures). It is apparent in Figs. 16a-d that consistent grid refinement provides a significant increase in the number of flow field structures resolved. In the prebreakdown region of the vortex core, substructures winding around the core are observed as the grid is refined.

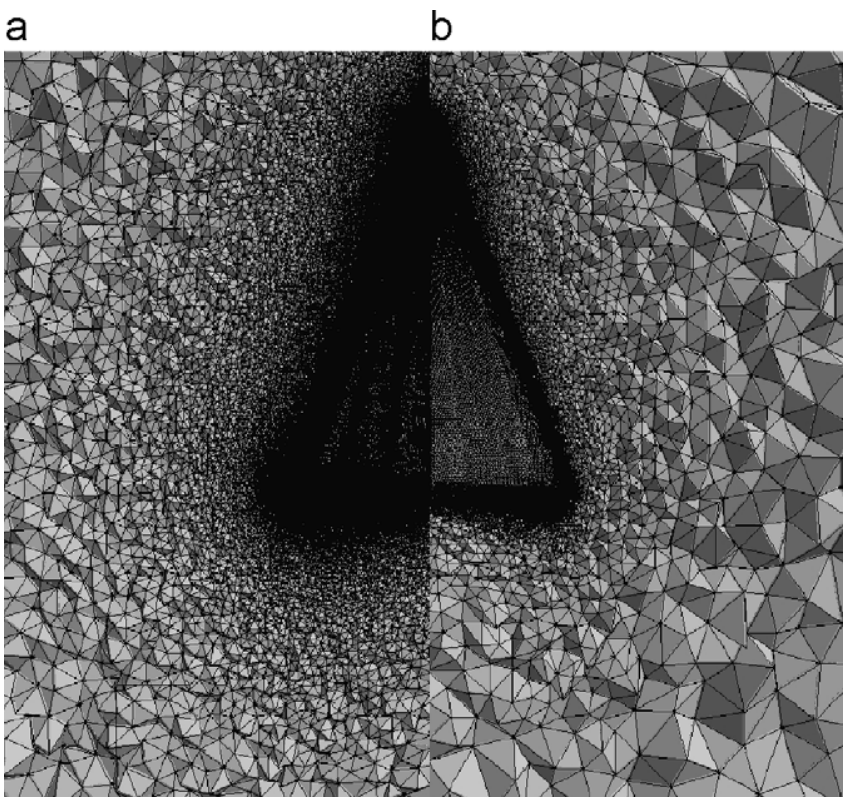

Fig. 15. Comparison of grids without and with AMR [19]: (a) grid 4 and (b) grid 5 (AMR).

Also, there is a significant increase in the number of structures observed in the region of the core, post-breakdown, as the grid is refined. Trailing-edge spanwise vortical structures begin to be resolved as the grid is refined, and for Grid 4, three-dimensional structures emanating from the blunt trailing edge that transit to spanwise coherent vortices are also captured. The trailing-edge coherent vortices also have an effect on the leading-edge shear layer, creating an instability at the leading edge that 

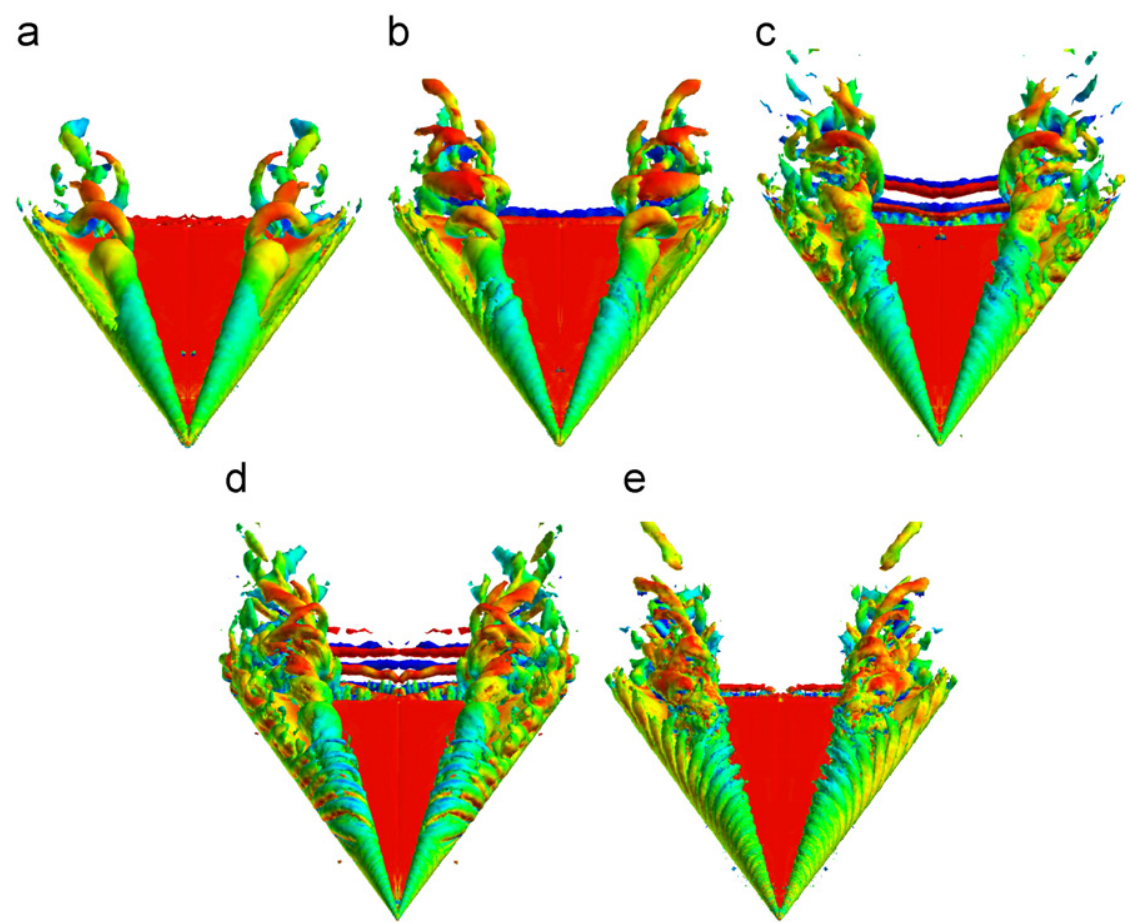

Fig. 16. Iso-surfaces of vorticity magnitude colored by spanwise vorticity; $\alpha=27^{\circ}, R e_{c}=4.5 \times 10^{6}$ [19]: (a) grid 1 ; (b) grid 2 ; (c) grid 3 ; (d) grid 4 and (e) grid 5 .

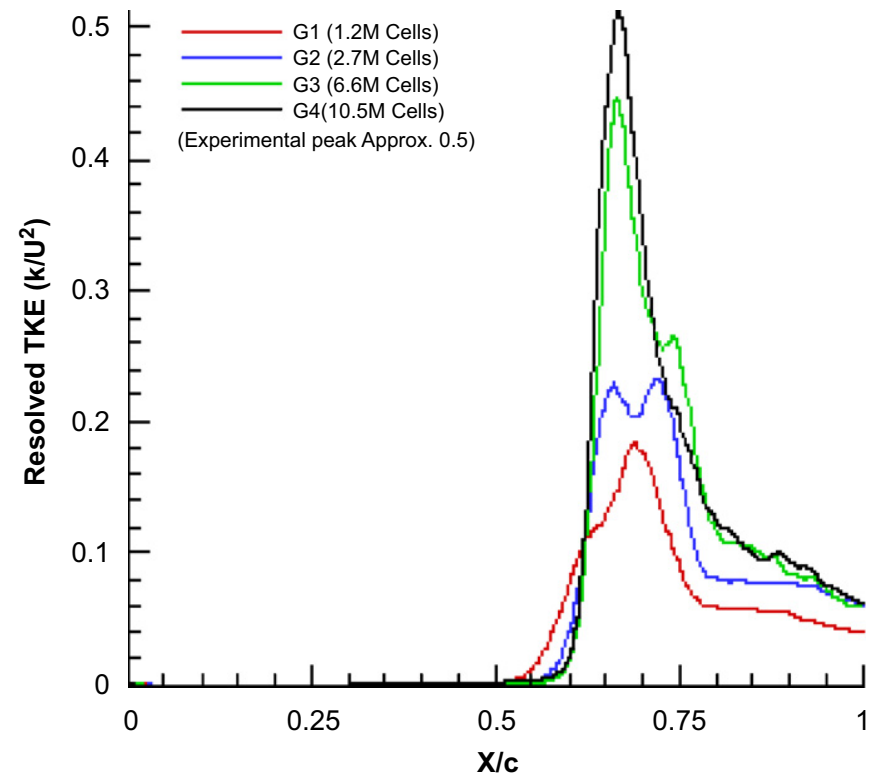

Fig. 17. Resolved turbulent kinetic energy non-dimensionalized by the square of the velocity along the core; $\alpha=27^{\circ}, R e_{c}=4.5 \times 10^{6}[19]$.

propagates forward as more of the trailing-edge vortices are resolved.

The AMR grid (Grid 5) depicted in Fig. 16e displays some significant differences in the pre-breakdown region. The vortical substructures are very coherent relative to the other grids and persist even downstream of the breakdown position. The trailing-edge vortices are evident but the coarseness of the grid in this region impedes the propaga- tion of these coherent structures downstream. The lack of leading-edge instability related to the trailing-edge coherent structures may be due to the decrease in shear layer resolution for Grid 5, post-breakdown, discussed above. Consistent with the fact that the core of the vortex is even more refined than Grid 4, there is a tremendous amount of three-dimensional structure in the region of the core, postbreakdown.

The resolved turbulent kinetic energy in the vortex core is shown in Fig. 17. Note that only Grid 4 was able to match the experimental peak of 0.5 . It should be noted that the AMR grid (Grid 5) also produced a peak turbulent kinetic energy of 0.5 , meaning that the grid with wellplaced cells in the vortex (Grid 5) was equivalent to the very fine grid (Grid 4) in this situation.

\section{Accurate simulation of full aircraft at flight Reynolds numbers}

In order to verify that the features we have discussed lead to accurate prediction of highly unsteady flows, we will show examples from three military aircraft flying at full-scale Reynolds numbers: the F-18C, F-16C, and F-16XL.

\subsection{High angle-of-attack flow over the F-18C}

Another important feature in the accurate prediction of time-dependent flows is the turbulence model used, as is demonstrated with computations done for the F-18C at $\alpha=30^{\circ}, M_{\infty}=\theta .2755$, and $R e_{c}=43.0 \times 10^{6}$. The halfbody mesh was created using AMR and the calculations 
were done with $\Delta t^{*}=\theta .012$, which is our baseline time step. For these conditions the F-18C experiences vortex breakdown over the wing, which causes highly unsteady, massively separated flow in the vicinity of the vertical tails, as shown in Fig. 18. These computations were reported in [21], including comparisons with flight-test data.

Three turbulence models were used to compute the flow over the F-18C: Shear stress transport (SST), SpalartAllmaras (SA), and Spalart-Allmaras with detached-eddy simulation (SADES). The lift and drag forces for these three turbulent models are shown in Fig. 19, and while the magnitudes for all three turbulence models are quite similar (within $0.1 \%$ of each other), the time variation is very different. The frequency content of the SADES contains a great deal more information, and we found that the frequencies on the vertical tail matched flight-test data when SADES was used [21].

The SADES solutions were used to perform a PSD analysis, and the results are presented in Fig. 20. While the power levels are different (due to a lack of knowledge about the reference conditions for the flight-test data), the SADES simulation accurately predicted the unsteady flow over the vertical tails for the aircraft.

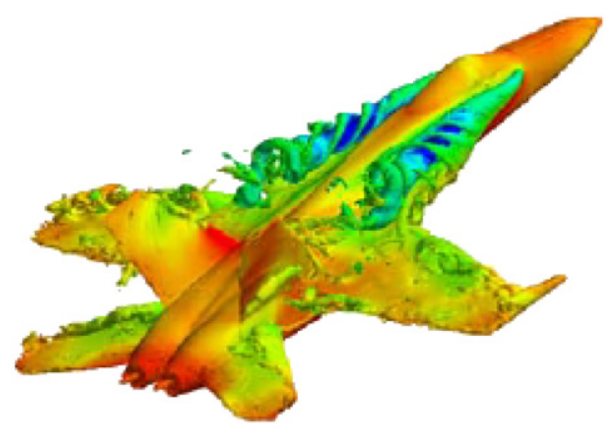

Fig. 18. F-18C with vortex breakdown over the wing; $\alpha=30^{\circ}$, $M_{\infty}=0.2755, R e_{c}=43.0 \times 10^{6}[21]$.

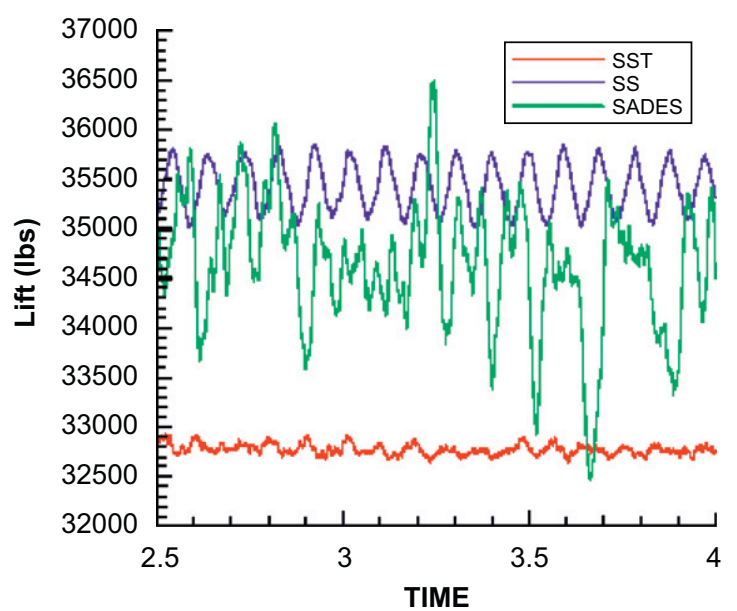

\subsection{High angle-of-attack flow over the F-16C}

One of the issues that was discussed earlier was the impact of temporal damping (or any type of artificial damping) on simulations that involved separated flow. Schiff et al. [8] showed that damping levels need to be reduced by several orders of magnitude before consistent results were obtained, and our experiences show that inappropriate levels of damping can have significant effects on separated flow predictions.

Flow over the F-16C was computed for $\alpha=\theta^{\circ}$, $M_{\infty}=\theta .6, R e_{c}=43.0 \times 10^{6}$ as a test case for the effects of temporal damping. Fig. 21 shows the damping effect, where

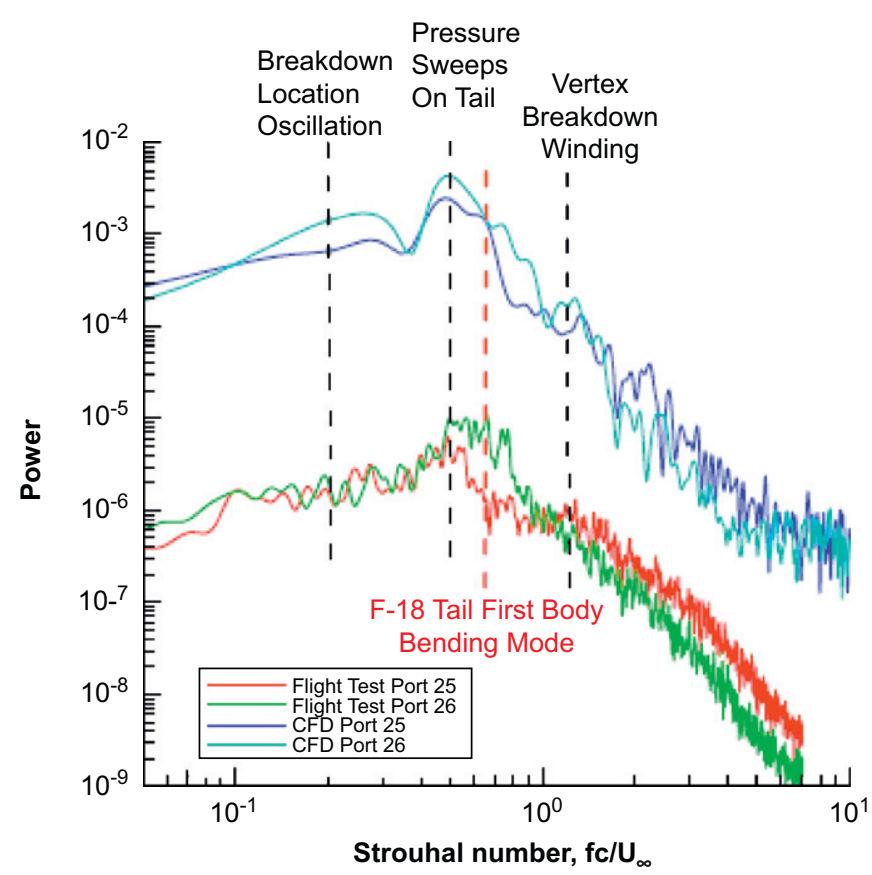

Fig. 20. Power spectrum density for vertical fins on F-18C; $\alpha=30^{\circ}$, $M_{\infty}=0.2755, \operatorname{Re}_{c}=43.0 \times 10^{6}[21]$.

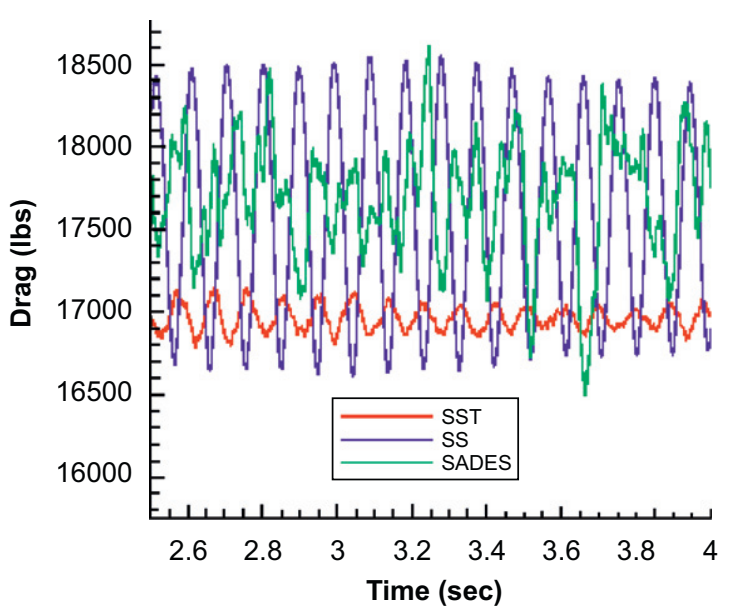

Fig. 19. Lift and drag variation for F-18C; SST = shear-stress transport, SA = Spalart-Allmaras, SADES = Spalart-Allmaras with detached-eddy simulation; $\alpha=30^{\circ}, M_{\infty}=0.2755, R e_{c}=43.0 \times 10^{6}[21]$. 


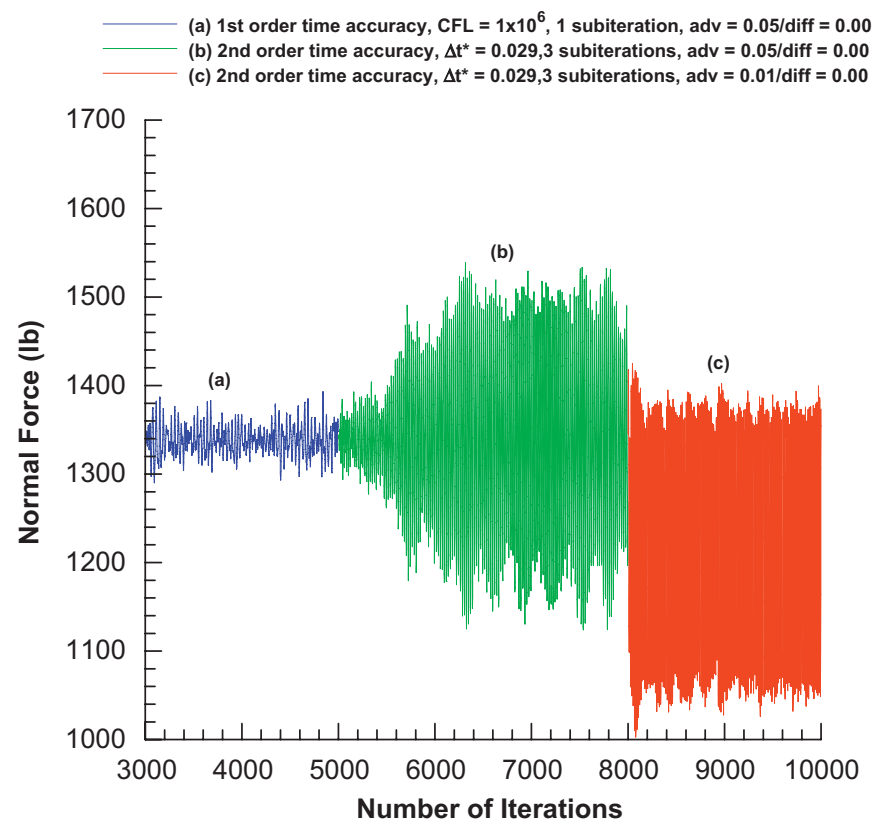

Fig. 21. F-16C normal force variation for steady and unsteady flow at one damping level, and for unsteady flow at a lower damping level; $\alpha=\theta^{\circ}$, $M_{\infty}=\theta .6, R e_{c}=43.0 \times 10^{6}$.

the advection damping has been changed from 0.05 (green, (b)) to 0.01 (red, (c)). The initial steady-state computations are shown in blue (a) and have a damping value of 0.05 . Note that after the damping level was decreased, the magnitude of the average normal force was decreased by approximately $10 \%$, a significant difference in force.

Another issue was discovered that showed how important it can be to appropriately choose the computational interval when looking at PSD or other statistical information. The insert graph in Fig. 22 shows the normal force variation for the F-16C at $\alpha=30^{\circ}, M_{\infty}=\theta .6, R e_{c}$ $=43.0 \times 10^{6}$, where it is apparent that the unsteady flow field has not been established until after several thousand iterations of start-up. The red line in the outer figure shows the average normal force based on the number of iterations used to take the average. The averages are taken starting with the last iteration computed and then averaging backward in time. The initial values oscillate quite a bit, as would be expected for an unsteady flow, but then the normal force settles down to a fairly constant level. Statistical information for the last 6000 iterations would probably yield reasonable results. However, if averages were taken that included the 6000-8000 iteration range (which would begin to include the start-up results for the simulation), a largely different result would be obtained. This may seem trivial, but shows the importance of using information only once an established flow is computed.

\subsection{Medium angle-of-attack flow over the F-16XL}

Calculations for the F-16XL were undertaken as part of the NATO RTO Task Group 113, which used the F-16XL

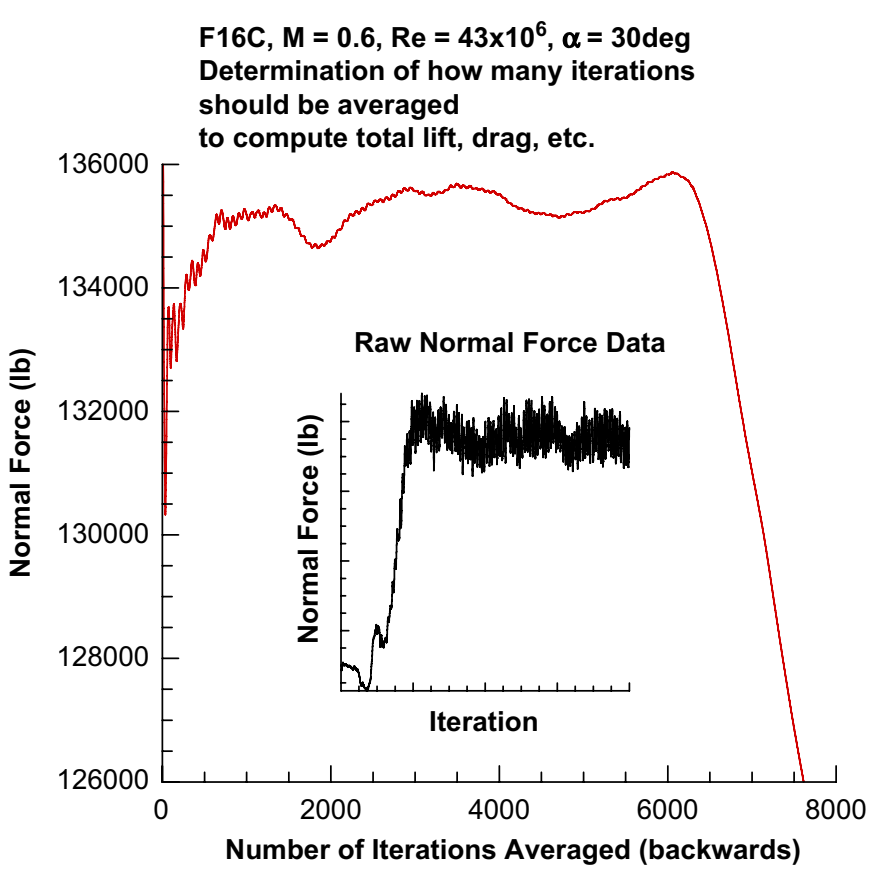

Fig. 22. Impact of simulation interval on time-averaged results.

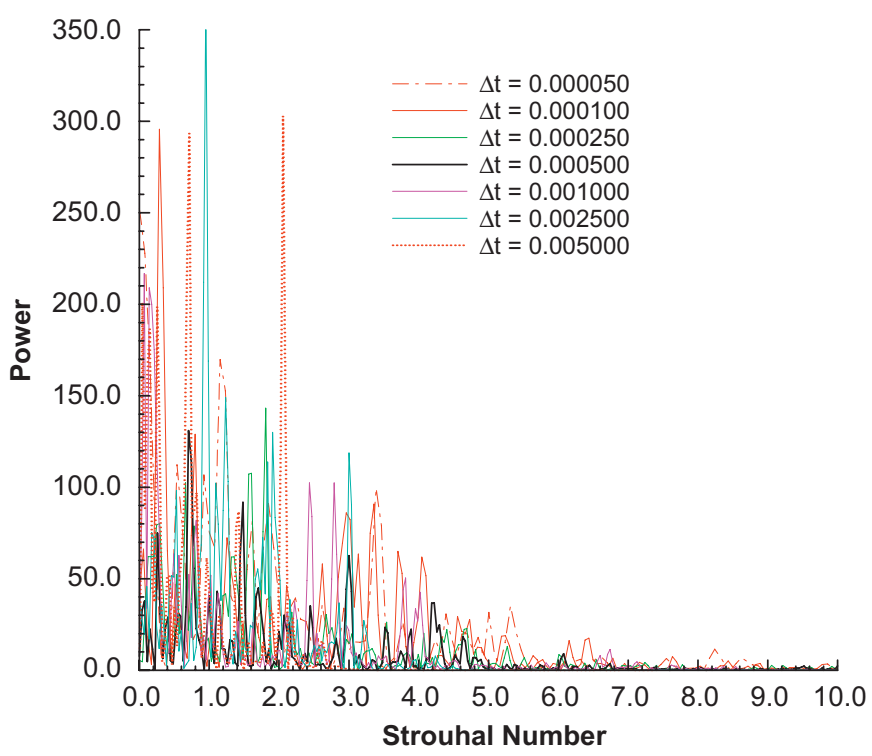

Fig. 23. F-16XL PSD for normal force, $\alpha=41.89^{\circ}, M_{\infty}=\theta .304$, $R e_{c}=44.4 \times 10^{6}$.

as a test bed for current CFD capabilities [22]. While performing the original time-step study, the aircraft normal force was used, resulting in the PSD analysis shown in Fig. 23. Note that there are no consistent results for this analysis: different time steps yield primary frequencies that do not form any discernable pattern. It became apparent that when looking at the full aircraft integrated forces (such as normal force), there were too many inputs into the resulting force. The integration process is probably 
masking important flow information from various locations on the aircraft.

Pressure "taps" were created in the flow field at various locations where pressure was saved for each iteration so that the PSD could be performed with localized information in the region of interest. Specifically, pressure taps were located in the region of unsteadiness for the leadingedge vortex, as shown by the white dots in Fig. 24.

The resulting PSD analysis is shown in Fig. 25a. There is now a consistent set of results, with the primary frequency "converging" as the time step is decreased. The primary frequencies are shown in Fig. 25b, which clearly shows that a non-dimensional time step of $\Delta t^{*}=\theta .01$ is probably adequate for obtaining physically realistic results in the leading-edge vortex region shown in Fig. 24. If other

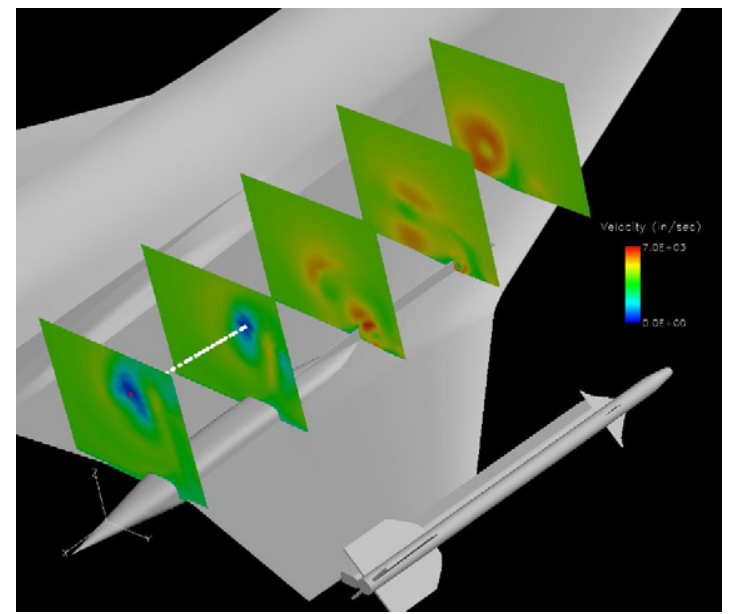

Fig. 24. F-16XL with pressure "taps" located in the region of interest (shown by white dots) [22].

a

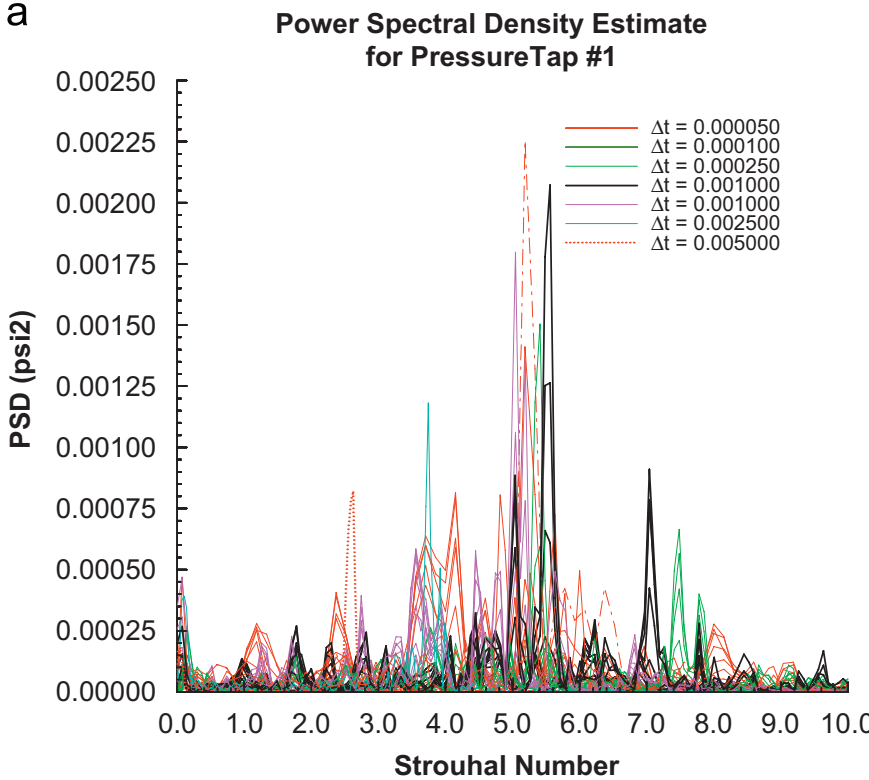

regions of the flow about the aircraft were also deemed important, then pressure taps could be located in those regions in order to expand the PSD study. In this way a single time step could be found that accurately resolved all of the flow features of interest.

\section{Proposed overall approach}

Obviously, the first step in our approach to accurately predict time-dependent flows is to create a grid for viscous calculations with appropriate grid spacing near the surface (turbulent flows should have $y^{+} \approx 1$, for example). We use the following theoretically based formulation to calculate the initial grid spacing, which seems to work very well for all Reynolds numbers we have investigated:

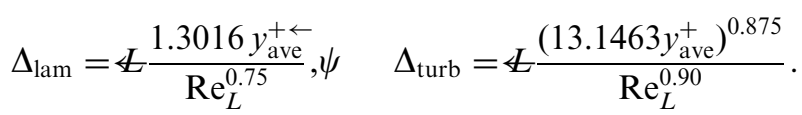

Once the initial grid spacing is determined, it should be insured that the growth rate away from the surface is not higher than approximately 1.25 , which should result in at least 20 grid points (or cell layers) in the boundary layer; this should be an adequate grid for a boundary layer. At least three grid levels should be created (coarse, medium, fine), where we usually use a $\sqrt{2}$ multiplication in all directions, which creates grids that have approximately a factor of 2 size difference (due to recombination of the viscous layer to create pyramids near the surface). Often additional grids may be required in order to obtain reasonable results, depending on the flow being simulated.

Now a time-step study on each grid has to be performed to see the impact of grid size and time step on the results, which assumes that the grids are reasonable to begin with.

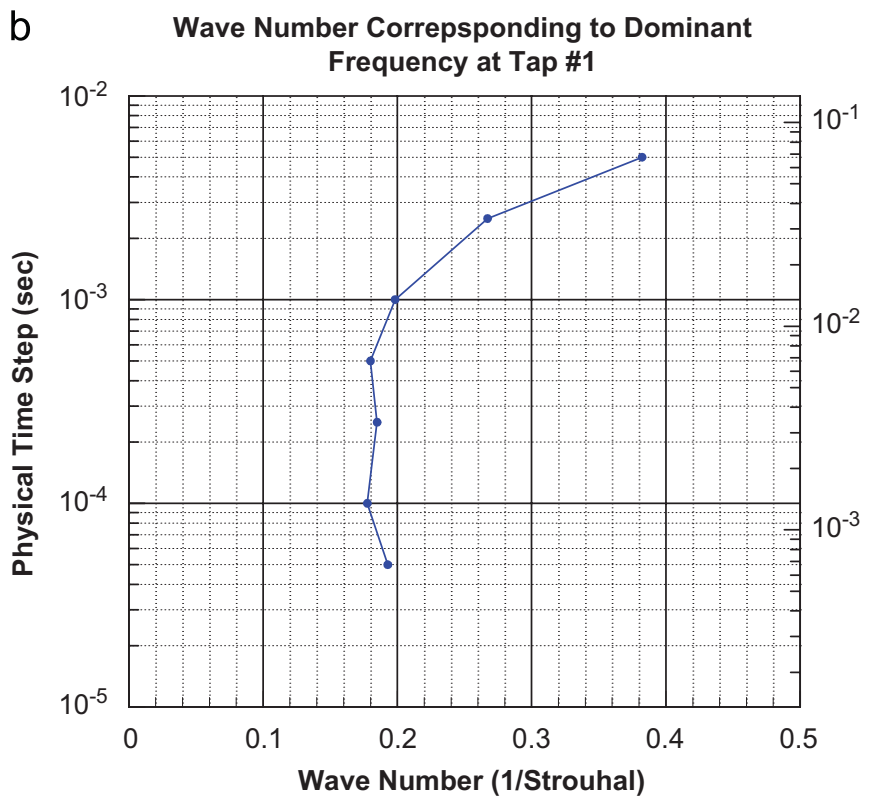

Fig. 25. F-16XL PSD for the first pressure tap and resulting wave number, $\alpha=41.89^{\circ}, M_{\infty}=\theta .304, R e_{c}=44.4 \times 10^{6}$ [21]: (a) PSD for 1st pressure tap and (b) wave number variation with time step. 


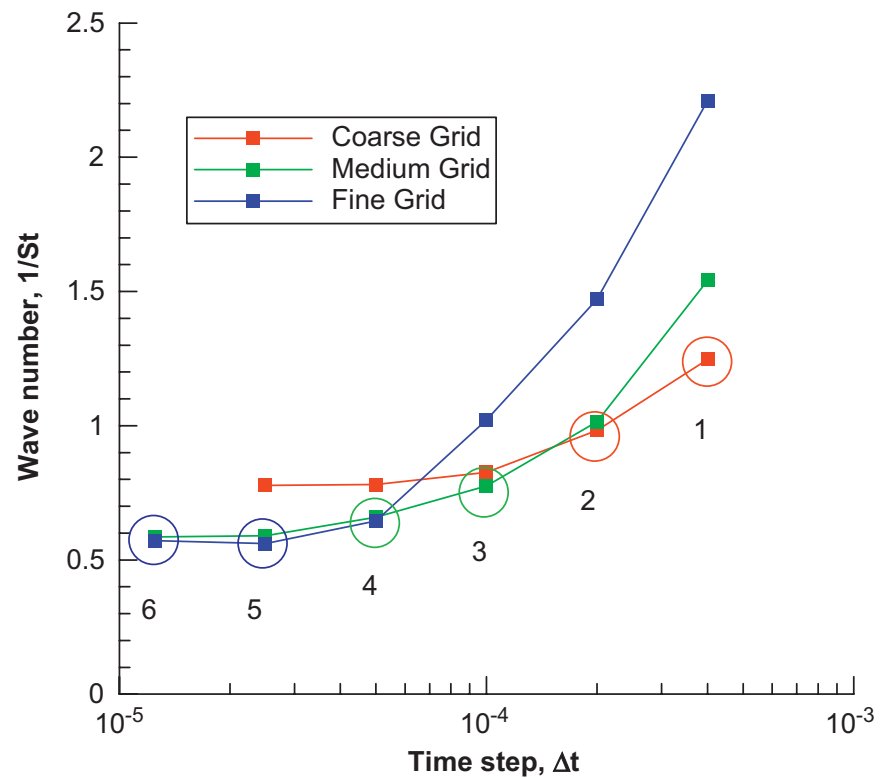

Fig. 26. Proposed poor man's "steepest descent" method for time-step study.

Unfortunately, this can be quite expensive, as has been shown with the various examples in this paper. The airfoil study results shown in Fig. 6 required $88 \mathrm{CPU}$ hours, running on a Linux cluster with eight processors for each case! A possible way to avoid this expense would be to use a poor man's "steepest descent" method, which takes advantage of the fact that our experience shows the wave number always decreases with decreasing time step until a "converged" time step is reached. This would allow for an approach as shown in Fig. 26 to be used, which uses the airfoil study results as a starting point. Using this approach, 15-18 converged solutions for three grids could be replaced by six solutions on three levels of grids at a lower computational cost (in this case, about $50 \mathrm{CPU}$ hours). Use $\Delta t^{*}=\theta .01$ (essentially the Nyquist sampling rate) as a starting point for the calculations: our results show that the correct time step is usually close to this value. Use time steps that are one-half order of magnitude larger and smaller than this value. Use DES for turbulent flow calculations with highly unsteady results. It is conceivable that some sort of optimization routine could be used to find the correct grid size and time step, but the additional difficulty and cost may not be worth the effort.

The overall approach shown in Fig. 26 could be summarized like this:

- Points 1 and 2: $\Delta t_{1}$ should be used on the coarse grid (Point 1) followed by another calculation with $\Delta t_{2}=\leftarrow$ $\Delta t_{1} / 2$ (Point 2);

- Points 3 and 4: $\Delta t_{3}=\Delta t_{2} / 2$ should be used on the medium grid (Point 3 ) followed by another calculation with $\Delta t_{4}=\Delta t_{3} / 2$ (Point 4 );

- Points 5 and 6: $\Delta t_{5}=\Delta t_{4} / 2$ should be used on the fine grid (Point 5) followed by another calculation with $\Delta t_{6}=\Delta t_{5} / 2$ (Point 6$)$
- If results from 4-6 are essentially the same, then the medium grid could be used to perform the calculations.

This would significantly reduce the computational time associated with finding the right grid and time step, but taking the time to perform these studies early on is worth the effort.

\section{Conclusions}

Results from several basic flow types as well as three fullscale aircraft have been presented for computing timedependent flows. A number of important lessons were learnt while performing these studies, including:

- 4nderstand what is important for your calculation and know the physics involved: the grid and time step should be determined by the flow region of interest;

- perform grid and time-step study in conjunction with one another;

- wary time step and number of iterations so that all computations are for the same physical time;

- eompute at least 10 cycles of the frequencies of interest;

- trse appropriate averaging over a reasonable simulation interval;

- perform PSD for frequency analysis;

- evaluate time-integration method (including sub-iterations) and damping for their impact on accuracy;

- 4tse the least amount of damping possible in simulations;

- trse taps instead of integrated forces if necessary for PSD;

- trse hybrid turbulence models if possible, including DES or DDES.

Our experience shows that good results can be obtained if this overall approach is taken, although there certainly could be other ways to insure that time-dependent flow computations are accurate.

\section{References}

[1] Lovell DA. Military vortices. In: RTO-MP-069, Paper KN-1, Proceedings of the NATO RTO/AVT symposium on advanced flow management, Loen, Norway, May 2001.

[2] Spalart PR, Jou W-H, Strelets M, Allmaras SR. Comments on the feasibility of LES for wings, and on a hybrid RANS/LES approach. In: Proceedings of the first AFOSR international conference on DNS/ LES, 4-8 August 1997. Ruston, LA: Greyden Press.

[3] Spalart PR, Deck S, Shur ML, Squires KD, Strelets MK, Travin A. A new version of detached-eddy simulation, resistant to ambiguous grid densities. Theor Comput Fluid Dyn 2006;20:181-95.

[4] Nyquist H. Certain factors affecting telegraph speed. Bell Syst Tech J 1924;3:324-46.

[5] Shannon CE. A mathematical theory of communication. Bell Syst Tech J 1948;27:373-423. 623-656.

[6] Alten SR. Audio in media. Belmont, CA: Wadsworth Publishing Co.; 1996.

[7] Spalart PR. Young person's guide to detached-eddy simulation grids. NASA CR 2001-211032, July 2001. 
[8] Schiff LB, Degani D, Cummings RM. Computation of three dimensional turbulent vortical flows on bodies at high incidence. J Aircr 1991;28(11):689-99.

[9] Strelets M. Detached eddy simulation of massively separated flows. In: AIAA Paper 2001-0879, Proceedings of the AIAA 39th aerospace sciences meeting, Reno, NV, January 2001.

[10] Görtz S. Detached-eddy simulations of a full-span delta wing at high incidence. In: AIAA Paper 2003-4216, Proceedings of the AIAA 21st applied aerodynamics conference, Orlando, FL, June 2003.

[11] Schiavetta L, Badcock K, Cummings RM. Comparison of DES and URANS for unsteady vortical flows over delta wings. In: AIAA Paper 2007-1085, Proceedings of the AIAA 45th aerospace sciences meeting, Reno, NV, January 2007.

[12] Strang WZ, Tomaro RF, Grismer MJ. The defining methods of Cobalt: a parallel, implicit, unstructured Euler/Navier-Stokes flow solver. In: AIAA Paper 99-0786, Proceedings of the AIAA 37th aerospace sciences meeting, Reno, NV, January 1999.

[13] Van Dyke M. An album of fluid motion. Stanford: Parabolic Press; 1982.

[14] Cebeci T, Smith AMO. Analysis of turbulent boundary layers. Orlando: Academic Press; 1974.

[15] Tennekes H, Lumley JL. A first course in turbulence. Cambridge, MA: The MIT Press; 1972.
[16] Ferziger JH, Peric M. Computational methods for fluid dynamics. New York: Springer; 1997.

[17] Huang RF, Lin CL. Vortex shedding and shear-layer instability of wing at low-Reynolds number. AIAA J 1995;33(8).

[18] Cummings RM, Morton SA, Siegel SG. Computational simulation and experimental measurements for a delta wing with periodic suction and blowing. J Aircr 2003;40(5):923-31.

[19] Mitchell AM, Morton SA, Forsythe JR, Cummings RM. Analysis of delta-wing vortical substructures using detached-eddy simulation. AIAA J 2006;44(5):964-72.

[20] Pirzadeh SZ. Vortical flow prediction using an adaptive unstructured grid method. In: NATO RTO MP-069, Paper SYA-13, Proceedings of the symposium on advanced flow management. Part A: vortex flows and high angle of attack for military vehicles, Loen, Norway, May 2001.

[21] Morton SA, Cummings RM, Kholodar D. High resolution turbulence treatment of F/A-18 tail buffet. J Aircr 2007;44: 1769-75.

[22] Morton SA, McDaniel DR, Cummings RM. F-16XL unsteady simulations for the CAWAPI facet of RTO Task Group AVT-113. In: AIAA Paper 2007-0493, Proceedings of the AIAA 45th aerospace sciences meeting, Reno, NV, January 2007. 